\title{
From Heisenberg matrix mechanics to semiclassical quantization: Theory and first applications
}

\author{
William R. Greenberg, Abraham Klein, and Ivaylo Zlatev \\ Department of Physics, University of Pennsylvania, Philadelphia, Pennsylvania 19104-6396 \\ Ching-Teh Li \\ Department of Physics, National Taiwan University, Tapai, Taiwan 10764, Republic of China
}

(Received 29 March 1996)

\begin{abstract}
Despite the seminal connection between classical multiply periodic motion and Heisenberg matrix mechanics and the massive amount of work done on the associated problem of semiclassical Einstein-Brillouin-Keller (EBK) quantization of bound states, we show that there are, nevertheless, a number of previously unexploited aspects of this relationship that bear on the quantum-classical correspondence. In particular, we emphasize a quantum variational principle that implies the classical variational principle for invariant tori. We also expose the more indirect connection between commutation relations and quantization of action variables. In the special case of a one-dimensional system a different and succinct algebraic derivation of the WKB quantization rule for bound states is given. With the help of several standard models with one or two degrees of freedom, we then illustrate how the methods of Heisenberg matrix mechanics described in this paper may be used to obtain quantum solutions with a modest increase in effort compared to semiclassical calculations. We also describe and apply a method for obtaining leading quantum corrections to EBK results. Finally, we suggest several modified applications of EBK quantization. [S1050-2947(96)06608-5]

PACS number(s): 03.65.Ca, 21.60.Ev, 21.60.Jz
\end{abstract}

\section{INTRODUCTION}

Though applications of great interest (and increasing complexity) continue to be developed, e.g., $[1,2]$, the theory of the semiclassical quantization of invariant tori by the application of Einstein-Brillouin-Keller (EBK) quantization conditions [3-5] appears to be a closed (or at least quiescent) sector in the study of the relationship between the quantum mechanics and the classical mechanics of nonseparable systems. (For exceptions, see the work of Jaffe and collaborators [6-8] and the even more recent work of Morehead [9].) Focusing on systems with two degrees of freedom, we can divide the many methods that have been developed and applied to this subject into two main subcategories those based on the solution of Hamilton's equations as an initial-value problem, i.e., the calculation of trajectories, and those based on algebraic methods involving trajectories indirectly or not at all. Within this subdivision, referred to as $M 1$ and $M 2$, respectively, we may distinguish principally the following.

Mla. Independent actions are computed from closed curves, signaling invariant tori, generated by the intersection of trajectories on two independent surfaces of section [1012].

$M 2 a$. If invariant tori exist, the dynamical variables can be represented as multiply periodic functions of the angle variables, with Fourier coefficients that depend only on the frequencies or, equivalently, actions. Equations of motion for the Fourier coefficients are obtained in either Hamiltonian or Lagrangian form and for each there is an associated variational principle. The equations of motion are solved either perturbatively or nonperturbatively and the quantized actions and energy calculated in terms of the known Fourier coefficients [13-21]. This is the approach of paramount interest to us.

$M 1 b$. Consider the system Hamiltonian to be a sum of an integrable part and a perturbation. The latter is turned on adiabatically over a time $T$. From the assumption that the actions computed initially for the unperturbed system are approximate adiabatic invariants, it is possible to obtain quantum energies $[22,23]$ (sometimes even for values of the coupling strength at which associated invariant tori no longer exist).

$M 2 b$. The Hamilton-Jacobi equation is solved iteratively, using a Fourier series expansion, thus providing the generating function for the appropriate action-angle variables [24]. (In contrast, the Fourier series constructed in $M 2 a$ are to be understood as the explicit equations of transformation from the original dynamical variables to the action-angle set.)

MIc. It can be shown $[25,26]$ that a finite set of adjacent nonquantized trajectories with the same total energy can be used to calculate accurate values of the actions for one of them. Quantized energies and associated actions are computed by linear extrapolation. In this method it is necessary to propagate trajectories until they almost close on themselves.

$M 2 c$. Following the ideas of Birkhoff and Gustavson [27], several groups [28-31] have carried out increasingly ambitious programs for transforming a given Hamiltonian to normal form by a succession of canonical transformations. The resulting Hamiltonian is quantized and energy values obtained.

M1d. Trajectories propagated over a sufficiently large multiple of the elementary periods of a multiply periodic orbit can be Fourier transformed to yield the Fourier representation of the fundamental dynamical variables. Applying Percival's formulas [17], the actions are computed. Initial conditions are varied until quantized orbits are found [3234].

For further discussion and a more exhaustive list of refer- 
ences, we refer the reader to Refs. [32,33,35].

Despite all this effort, there remains a gap in the study of the relationship between quantum mechanics and the theory of invariant tori, especially as it relates to the work that utilizes the description of invariant tori in terms of Fourier series. This assertion is grounded on the observation that each form of quantum mechanics is associated in a natural way with a corresponding version of classical mechanics. Thus, in the same sense that Schrödinger wave mechanics is naturally associated with the Hamilton-Jacobi equation and the classical limit of the path-integral method is Hamilton's variational principle, it is equally true that the classical limit of Heisenberg matrix mechanics for bound systems are the equations of motion for multiply periodic systems. Though some of the work cited is based, in the sense of the correspondence principle, on this passage between quantum and classical descriptions and the passage itself is in some respects well known (see [6-8], for instance), the main thesis of this paper is that this route for passing from the quantum to classical theory has not been fully explored. A possible explanation is that, although the basic correspondence is well known, it is hardly known outside the circle of the authors and former associates that matrix mechanics can be derived from a different variational principle [36-39] called the trace variational principle (see also [40]) and that the classical limit of this principle is the variational principle for invariant tori [21].

Guided by this relationship, the main purpose of this work is to study the transition from the quantum to the classical domain more thoroughly than heretofore for both the dynamics and the kinematics (quantization conditions). This has led to some results that we find it difficult to believe are not known, but for which we do not have an independent reference. In Sec. II we supply a brief but self-contained account of our version of matrix mechanics, with emphasis on the variational basis. The passage to the semiclassical limit is then carried out in Secs. III and IV, where we show that the limit of the quantum variational principle is the variational principle for invariant tori. We find, however, that the relationship between the commutation relations and the EBK quantization conditions is more indirect, the former corresponding in the classical limit to the Poisson bracket relations and the latter to the Lagrange bracket relations [41]. In Sec. V we study the commutation relations per se, the most tangible result being another derivation of the WKB quantization condition, applicable to one-dimensional systems. Section VI is devoted to some illustrative numerical studies. We describe in turn and then apply algorithms for carrying out the semiclassical calculations, the associated matrix quantum calculations, and a method for calculating directly quantum corrections to the semiclassical result. The need for the latter as a separate approach arises from the fact that the matrix quantum calculations are designed to give better results than the semiclassical one for low-lying states, but do not go over in any limit to the EBK calculation. Finally, Sec. VII contains several suggestions for alternative ways to use the semiclassical quantization scheme. In Sec. VIII, we make some proposals for further work. Preliminary accounts of the main theoretical results of this paper can be found in $[42,43]$.

\section{MATRIX MECHANICS}

\section{A. Variational principles and equations of motion}

We begin with a brief description of a variational principle, the so-called trace variational principle, from which one can derive Heisenberg's form of quantum mechanics. Though a version of this principle was suggested more than three decades ago [36] and subsequently several publications have been devoted to its exposition and further development [37-39], it appears to be largely unknown by the community at large. Except for one brief allusion, [21], it has not been applied to the problem at hand.

Most of the important elements are already present for a system with one degree of freedom and we therefore focus attention on the Hamiltonian

$$
H=\frac{1}{2} p^{2}+V(x)
$$

with equations of motion

$$
\begin{gathered}
{[x, H]=i p,} \\
{[i p, H]=d V / d x \equiv V^{\prime},}
\end{gathered}
$$

derived by utilization of the commutation relation $(\hbar=1)$

$$
[x, p]=i .
$$

In practice we are usually concerned with the matrix elements of (2.2)-(2.4) in the representation in which $H$ is diagonal, with eigenvalues $E_{n}$, namely,

$$
\begin{gathered}
\left(E_{n}-E_{m}\right) x_{m n}=i p_{m n}, \\
\left(E_{n}-E_{m}\right) i p_{m n}=\left(V^{\prime}\right)_{m n},
\end{gathered}
$$

and

$$
[x, p]_{n m}=i \delta_{n m},
$$

where $x_{n m}=\langle n|x| m\rangle$. We shall feel free to use both notations interchangeably.

In early work [44] we have shown, for polynomial potentials, how the energy differences and the matrix elements $x_{m n}, p_{m n}$ can be obtained from Eqs. (2.5) and (2.6). The primacy of these elements can be seen by using the completeness relation for the evaluation of matrix elements of a product. The eigenvalues themselves can be found by the direct evaluation of the expectation values

$E_{n}=H_{n n}=\langle n|H| n\rangle=\sum_{n^{\prime}} \frac{1}{2}\left|p_{n n^{\prime}}\right|^{2}+\langle n|V(x)| n\rangle$,

where the application of completeness is illustrated in the kinetic-energy term. Ultimately, we shall be concerned with extending the previous algorithms to the multidimensional case.

Before proceeding to the discussion of a variational formulation, we add a few remarks about the implementation of the above formalism. Equations (2.5) and (2.6) are, to start with, an infinite set of sum rules that must be satisfied by the exact eigenstates of the Hamiltonian. Starting at any point in 
the spectrum, sums spread without cutoff as far as the configuration space will allow. To obtain closure, we must make two kinds of approximation. The first is that the matrix elements are rapidly decreasing functions of $k=|n-m|$, so that all matrix elements with $k>k_{\max }$ can be set to zero. The second is that the matrix elements are, for sufficiently large $n$ and $m$, slowly varying functions of $n+m$, so that for some values sufficiently far from the center of interest in a given calculation we can set $\langle n+r|x| m+r\rangle=\langle n|x| m\rangle$ for $|r|$ $\ll \frac{1}{2}(m+n)$. With these assumptions Eqs. (2.5) and (2.6) reduce to a finite set of equations, where in addition to the retained matrix elements of $x$ and $p$, the additional unknowns are a set of elementary energy differences from which all other energy differences can be composed. To determine this collection of variables, it suffices to utilize all the available equations of motion, but only the diagonal elements of the commutator. This is consistent with the result, proved in Sec. II B, that the off-diagonal elements of the commutator are a consequence of the equations of motion.

A natural question to ask is whether Eqs. (2.5) can be derived from a variational principle. Here we wish to treat the matrix elements of $x$ and $p$ as variables in the variational statement $\delta E_{n}=\delta H_{n n}=0$. There are, however, two obstacles to such an endeavor: (i) The matrix elements are not all independent and (ii) the same matrix elements appear in different energy functionals. Thus $p_{n n^{\prime}}$ occurs both in $H_{n n}$ and in $H_{n^{\prime} n^{\prime}}$. For which is it to be a variational parameter? A solution to the second problem posed is to form an average of the stationary functionals. It turns out that in order to derive the equations of motion as given above, it is necessary to choose the most symmetrical possible average, namely, the trace. Thus we require

$$
\delta \sum_{n} H_{n n}=\delta \operatorname{Tr} H=0 .
$$

A solution to the first problem is to impose all the possible kinematical constraints, namely,

$$
\delta[x, p]_{n n^{\prime}}=0
$$

Multiplying (2.9) by a Lagrange multiplier matrix ( $-i) \Lambda_{n^{\prime} n}$ ( $\Lambda$ is Hermitian), we add the result to (2.8) and are thus led to a master variational principle

$$
\begin{aligned}
0 & =\delta F \equiv \delta \operatorname{Tr}\{H-i \Lambda[x, p]\} \\
& =\delta \operatorname{Tr}\{H-i p[\Lambda, x]\}=\delta \operatorname{Tr}\{H+i x[\Lambda, p]\} .
\end{aligned}
$$

The several forms are equivalent because of the assumed cyclic invariance of the trace. (This is certainly unobjectionable in practice where the trace is taken over a finitedimensional vector space.)

Carrying out the unconstrained variation of (2.10) with respect to the matrix elements $x_{n^{\prime} n}$ and $p_{n^{\prime} n}$, keeping $\Lambda$ fixed, and using the explicit form (2.1) of $H$ we obtain the equations

$$
\begin{aligned}
& p_{n^{\prime} n}=-i[x, \Lambda]_{n n^{\prime}}, \\
& \left(V^{\prime}\right)_{n n^{\prime}}=i[p, \Lambda]_{n n^{\prime}}
\end{aligned}
$$

Because of the invariance of the trace with respect to choice of basis, the representation $|n\rangle$ is, at this point, arbitrary. The most convenient immediate choice is the one in which the Hermitian operator $\Lambda$ is diagonal. By comparing with the known equations of motion, we then identify $\Lambda$ as the Hamiltonian.

The derivation of the equations of motion does not exhaust the consequences that can be drawn from the trace variational principle. We shall now demonstrate from this principle that the vanishing of the off-diagonal matrix elements of the canonical commutator is a consequence of the equations of motion, leaving only the diagonal elements as independent kinematical conditions. This result is consistent with the "empirical" finding above that a dynamical scheme is, in fact, completely determined by adjoining this onedimensional (diagonal) array of kinematical constraints to the equations of motion.

To derive the off-diagonal elements of (2.6), we make use of the invariance of the trace under an infinitesimal change of basis. In the new basis, the Hamiltonian will not be diagonal, in general, and thus we must allow for a change in the Lagrange multiplier matrix. We calculate

$$
0=\delta F=\operatorname{Tr}\{-i(\delta H)[x, p]\},
$$

since all other contributions vanish as a consequence of the equations of motion. If we express the infinitesimal change of basis in the standard form

$$
\delta|n\rangle=-i \epsilon \Theta|n\rangle
$$

where $\epsilon$ is infinitesimal and $\Theta$ is Hermitian, we recognize that in a variation about the energy diagonal representation

$$
\delta\langle n|H| n\rangle=i \epsilon\langle n|[\Theta, H]| n\rangle=0 .
$$

On the other hand, nondiagonal elements

$$
\delta\langle n|H| m\rangle \equiv\langle n|\delta H| m\rangle \equiv \delta H_{n m}
$$

can be assigned arbitrary infinitesimal values consistent with Hermiticity. From this and (2.13) we conclude that the offdiagonal elements of $[x, p]$ vanish.

One additional result of great importance is that a solution of the dynamical scheme proposed above guarantees that the Hamiltonian is diagonal, $H_{n, n^{\prime}}=0, n \neq n^{\prime}$. This result is derived in the Appendix.

In place of Hamilton's equations (2.5) it is often more convenient to consider Newton's equation

$$
\left(E_{n}-E_{m}\right)^{2} x_{m n}=\left(V^{\prime}\right)_{m n} .
$$

This equation may be derived from its own variational principle by substituting the first of Eqs. (2.5) into the previous functional $F$. The result is a new functional $G$ that can be written in the alternative forms

$$
G \equiv \operatorname{Tr}\{H-H[x,[H, x]]\}=\operatorname{Tr}\left\{-\frac{1}{2}[x, H][H, x]+V(x)\right\} .
$$

In the second form, we recognize that $G$ is the negative of the trace of the Lagrangian. To obtain Newton's (or Lagrange's) equations one varies $G$ with respect to the ma- 
trix elements of $x$, keeping the matrix elements of the Hamiltonian fixed. From the structure of (2.18), this means keeping the energy differences fixed. This formulation of the quantum theory is completed by adjoining a form of the canonical commutator from which the momentum operator has also been eliminated, namely,

$\delta_{n m}=[x,[H, x]]_{n m}=\sum_{l}\left(2 E_{l}-E_{m}-E_{n}\right) x_{m l} x_{l n}$.

It should also be mentioned that the vanishing of the offdiagonal matrix element of the commutator can equally well be proved by paraphrasing for the functional $G$ the argument presented for the functional $F$.

Since our main interest in this paper is in autonomous systems with at least two degrees of freedom, we must now describe how the previous considerations are modified by this generalization. We therefore consider a system with $N$ coordinates $\mathbf{x}=\left(x_{1}, \ldots, x_{N}\right)$ and a Hamiltonian of the form

$$
H=\sum_{i} \frac{1}{2} p_{i}^{2}+V(\mathbf{x})
$$

The functional $F$ from which Hamilton's equations are derived takes the form

$$
F=\operatorname{Tr}\left\{H-i \Lambda \sum_{i}\left[x_{i}, p_{i}\right]\right\},
$$

where $\Lambda$ is once again identified as the Hamiltonian. Lagrange's equations are derived from the functional $G$, where

$$
G=\operatorname{Tr}\left\{-\frac{1}{2} \sum_{i}\left[x_{i}, H\right]\left[H, x_{i}\right]+V(\mathbf{x})\right\} .
$$

Before providing any further details, we must mention the problem of labeling the eigenvalues and eigenstates of $H$. In our earlier work [21], we "naturally" assumed that the labeling could be done by a choice of $N$ integers $\mathbf{n}=\left(n_{1}, \ldots, n_{N}\right)$ of which we could keep track as we tuned one or more coupling parameters, starting from values for which the problem was integrable. The same assumption was discussed rather more thoroughly by Percival [14-16], who emphasized that the validity of this assumption is coterminous, in the semiclassical limit, with the existence of invariant tori.

With this understanding, it is not necessary to write the equations of motion a second time, but only to remember that there is now one equation for each value of $i$ and in each of these equations to replace the integer $n$ by the corresponding vector $\mathbf{n}$. There does remain one question to be addressed that will be of some importance to us later. This is the question of whether we can deduce from the variational principle the separate vanishing, for each value of $i$, of the offdiagonal elements of the commutators $\left[x_{i}, p_{i}\right]$. This follows from the fact that the rows and columns of the matrix $\Lambda$ are each labeled by an $N$-dimensional vector, provided that the set of nonvanishing matrix elements of the product $x_{i} p_{i}$ is disjoint from the corresponding set for any other choice of coordinate index. This will be true for any model that we study. We have been somewhat cavalier in the present discussion, but the questions that we have slighted, in particular, why other elements of the algebra do not appear in the constrained variational principle, will be considered in more detail in the discussion that now follows.

\section{B. Commutation relations and equations of motion}

The canonical commutation relations must be constants of the motion; this means that their time derivatives should vanish [45]. We show this for the class of Hamiltonians under study. Consider first

$$
\frac{d}{d t}\left[p_{i}, p_{j}\right]=-\left[\frac{\partial V}{\partial x_{i}}, p_{j}\right]-\left[p_{i}, \frac{\partial V}{\partial x_{j}}\right]=0 .
$$

This calculation shows that in the energy-diagonal representation (assuming no degeneracy) the commutator of two different components of momenta has no off-diagonal matrix elements. If we choose the momenta to be imaginary Hermitian operators, it follows that the diagonal elements of the commutator also vanish. Thus it is clear that the commutators $\left[p_{i}, p_{j}\right]=0$ may be omitted from the dynamical scheme.

Utilizing the previous result that the off-diagonal elements of the commutator $\left[x_{i}, p_{i}\right]$ vanish, we calculate

$$
0=\frac{d}{d t}\left[x_{i}, p_{i}\right]=\left[x_{i}, \frac{\partial V}{\partial x_{i}}\right] .
$$

Since $V$ is to a large extent arbitrary, we may safely conclude that the coordinates all commute with one another.

Combining the previous two results, we next verify that

$$
\frac{d}{d t}\left[x_{i}, p_{j}\right]=\left[p_{i}, p_{j}\right]+\left[x_{i}, \frac{\partial V}{\partial x_{j}}\right]=0 .
$$

Finally, we check compatibility by the calculation

$$
\frac{d}{d t}\left[x_{i}, x_{j}\right]=\left[x_{i}, p_{j}\right]+\left[p_{i}, x_{j}\right]=0 .
$$

We have thus shown that the commutation relations are compatible with the equations of motion for the class of Hamiltonians under consideration. For the practical problem of constructing a calculus based on Heisenberg matrix mechanics, the consequence of our deliberations is that at most only the elements diagonal in the energy representation of the commutators of a coordinate and the corresponding momentum can enter, if we use all the available equations of motion.

\section{SEMICLASSICAL LIMIT: MATHEMATICAL PRELIMINARIES}

The purpose of this section and the one to follow is to show that the semiclassical theory of invariant tori is the "natural" limit of the quantum theory of the preceding section. We shall first collect in the form of lemmas some of the mathematical statements that we need. In the following we shall use the notation $\lim \langle\mathbf{n}|O| \mathbf{n}\rangle$ to signify the leading term in the semiclassical limit of the designated matrix element. Here $O$ is generally a product of elementary operators. We 
consider first the one-dimensional case and define for a real Hermitian operator $A$,

$$
A_{k}(n) \equiv\left\langle n-\frac{1}{2} k|A| n+\frac{1}{2} k\right\rangle=A_{-k}(n) .
$$

Notice that this quantity is an analytic continuation of a nearby physical matrix element and is our definition of the semiclassical limit of the matrix element $\langle n|A| n+k\rangle$. As shown in a previous work [20], this choice can be used to provide a completely algebraic basis for the standard WKB quantization rule for a vibrational degree of freedom.

We note that with the definition (3.1), we have, for the first two terms of a Taylor expansion,

$$
\langle n|A| n \pm k\rangle \cong A_{k}(n) \pm \frac{1}{2} k \frac{\partial A_{k}(n)}{\partial n} .
$$

Next, with the array of amplitudes $A_{k}(n)$, for fixed $n$ and varying $k$ we associate a formal Fourier series

$$
A(n, \theta)=\sum_{k=-\infty}^{\infty} A_{k}(n) \exp (i k \theta),
$$

though in practice we shall always deal with severely restricted sums. Below, we shall then make extensive use of the average

$$
\langle A B C \cdots\rangle \equiv(2 \pi)^{-1} \int d \theta A(n, \theta) B(n, \theta) C(n, \theta) \cdots,
$$

which is just the constant term in the Fourier series of the product.

With the above preliminaries, we are prepared to state and prove a series of elementary propositions.

Lemma 1:

$$
\lim \langle n|A B| n\rangle=\langle A B\rangle .
$$

For the proof we write

$$
\begin{aligned}
\lim \langle n|A B| n\rangle= & \lim \sum_{k>0}^{k_{\max }}[\langle n|A| n+k\rangle\langle n+k|B| n\rangle \\
& +\langle n|A| n-k\rangle\langle n-k|B| n\rangle] .
\end{aligned}
$$

Here we have assumed that $n$ is sufficiently large that the sum over $k$ can be extended far enough in both directions, to $k_{\max }$, to obtain numerical convergence. This establishes the limited and nonrigorous sense in which the word proof is to be understood both here and below.

If we now apply (3.2) in sequence first to the matrix elements of $A$, for example, and subsequently to the matrix elements of $B$, we find

$$
\begin{aligned}
\lim \langle n|A B| n\rangle & =\sum_{\text {all } k} A_{k}(n) B_{-k}(n)\left[1+O\left(n^{-2}\right)\right] \\
& =\langle A B\rangle\left[1+O\left(n^{-2}\right)\right] .
\end{aligned}
$$

The error estimate arises from the assumption that a derivative with respect to $n$ is of relative order $(1 / n)$. That the error is of second order is a consequence of our choice of definition (3.1). The sum on the right-hand side of (3.7) has the value required by the lemma.

Lemma $1(a)$. With the same assumptions as before, Lemma 1 can be extended to a product of more than two factors,

$$
\begin{aligned}
& \lim \langle n|A B C \cdots| n\rangle=\langle A B C \cdots\rangle \\
& \quad=\sum \delta_{k_{1}+k_{2}+k_{3}+\cdots, 0} A_{k_{1}}(n) B_{k_{2}}(n) C_{k_{3}}(n) \cdots .
\end{aligned}
$$

The proof is carried out by ordering the various upwardgoing, downward-going, and mixed contributions to the multiple sum so that Eq. (3.2) can be applied.

Lemma $1(b)$. The previous lemmas can be extended to the multidimensional case. Extending the boldface notation now to designate, in addition to the quantum numbers $\mathbf{n}$, also the integer vector $\mathbf{k}$ for the components of a multiple Fourier series and the vector $\boldsymbol{\theta}$ for an array of angle variables, we introduce a formal multiple Fourier series

$$
A(\mathbf{n}, \boldsymbol{\theta})=\sum_{\mathbf{k}} A_{\mathbf{k}}(\mathbf{n}) \exp (i \mathbf{k} \cdot \boldsymbol{\theta}) .
$$

The lemma then applies to the average

$$
\langle A B\rangle \equiv(2 \pi)^{-N} \int d \boldsymbol{\theta} A(\mathbf{n}, \boldsymbol{\theta}) B(\mathbf{n}, \boldsymbol{\theta})=\sum_{\text {all } \mathbf{k}} A_{\mathbf{k}}(\mathbf{n}) B_{-\mathbf{k}}(\mathbf{n})
$$

and to corresponding multiple products.

Lemma 2:

$$
\lim \langle n|O| n+k\rangle=O_{k}(n)=(2 \pi)^{-1} \int d \theta \exp (-i k \theta) O(n, \theta) .
$$

Here $O$ is a product of two or more elementary operators, since for a single operator the previous statement is only a combination of the definitions (3.1) and (3.3). The same equation applies in boldface notation. We shall not actually need this lemma, since its application would be to obtain the semiclassical limit of the equations of motion directly. Our procedure, however, will be to obtain that limit for the variational principle and then derive the equations of motion from the latter.

We do require expressions for limits of commutators. We consider the usual Poisson bracket

$$
[A, B]_{\mathrm{PB}} \equiv \sum_{i}\left[\frac{\partial A(\mathbf{n}, \boldsymbol{\theta})}{\partial \theta_{i}} \frac{\partial B(\mathbf{n}, \boldsymbol{\theta})}{\partial n_{i}}-\frac{\partial A(\mathbf{n}, \boldsymbol{\theta})}{\partial n_{i}} \frac{\partial B(\mathbf{n}, \boldsymbol{\theta})}{\partial \theta_{i}}\right] .
$$

We can now state the following.

Lemma 3:

$$
\lim \langle\mathbf{n}|[A, B]| \mathbf{n}+\mathbf{k}\rangle=i\left\langle[A, B]_{\mathrm{PB}}\right\rangle_{\mathbf{k}},
$$

where the notion on the right-hand side is understood as the kth Fourier component of the Poisson bracket (3.12). This 
lemma can be extended to multiple commutators. In practice, we shall only require the double commutator, for which we find the following.

Lemma 3(a):

$\lim \langle\mathbf{n}|[A,[B, C]]| \mathbf{n}+\mathbf{k}\rangle=(i)^{2}\left\langle\left[A,[B, C]_{\mathrm{PB}}\right]_{\mathrm{PB}}\right\rangle_{\mathbf{k}}$.

We shall utilize these results to develop the dynamics and kinematics of a semiclassical quantization scheme. Before turning to this task, we note an important consequence of the results in this section. Whereas in quantities such as $\langle\mathbf{n}|A B C| \mathbf{n}\rangle$ different base values of $\mathbf{n}$ will occur, it is a consequence of the elementary reasoning applied above that in the expression $\lim \langle\mathbf{n}|A B C| \mathbf{n}\rangle$ only the reference value of $\mathbf{n}$ occurs. Thus, in the semiclassical limit a trace decomposes into a sum of independent terms.

\section{SEMICLASSICAL LIMIT: DYNAMICAL AND KINEMATICAL SCHEME}

\section{A. EBK scheme}

We now apply the reasoning and results of the preceding section to obtain the semiclassical limit of the (Lagrangian) form of the trace variational principle. We are interested in a value of the vector $\mathbf{n}$ for which the corresponding energy and eigenstate may be described accurately by the semiclassical approximation. We should then form the trace over a space of states extending to both smaller and larger values of $\mathbf{n}$ compared to the reference state. From the results of the preceding section, however, it follows that for the purpose of deriving equations of motion we may suppress the trace, because in the limit considered, as we have already pointed out and in contrast to the quantum case, the states decouple. It thus suffices to focus attention on a given state. (At the end of this section, however, we shall have occasion to restore the trace.)

We consider first the kinetic energy $T$. If $E(\mathbf{n})$ is the energy of the state identified by $\mathbf{n}$, then here we shall encounter the correspondence principle in the form

$$
\begin{aligned}
\omega_{i}(\mathbf{n}) & \equiv E\left(n_{1}, \ldots, n_{i}+\frac{1}{2}, \ldots,\right)-E\left(n_{1}, \ldots, n_{i}-\frac{1}{2}, \ldots,\right) \\
& \cong\left[\partial E(\mathbf{n}) / \partial n_{i}\right]
\end{aligned}
$$

i.e., the classical frequencies $\boldsymbol{\omega}(\mathbf{n})=\left(\omega_{1}, \ldots, \omega_{N}\right)$ approach the quantum energy differences. We then find by a perfectly straightforward examination that

$$
\begin{aligned}
\lim 2\langle\mathbf{n}|T| \mathbf{n}\rangle= & \lim \sum_{i}\left\langle\mathbf{n}\left|\left[x_{i}, H\right]\left[H, x_{i}\right]\right| \mathbf{n}\right\rangle \\
= & \lim \sum_{i, \mathbf{k}}[E(\mathbf{n}+\mathbf{k})-E(\mathbf{n})]^{2} \\
& \times\left\langle\mathbf{n}\left|x_{i}\right| \mathbf{n}+\mathbf{k}\right\rangle\left\langle\mathbf{n}+\mathbf{k}\left|x_{i}\right| \mathbf{n}\right\rangle \\
= & \sum_{i, \mathbf{k}}(\mathbf{k} \cdot \boldsymbol{\omega})^{2} x_{i, \mathbf{k}}(\mathbf{n}) x_{i,-\mathbf{k}}(\mathbf{n})=\langle 2 T\rangle .
\end{aligned}
$$

We note that the same result may be found by applying Lemma 3 for the classical limit of a Fourier component of a commutator, provided that we recognize that the classical
Hamiltonian is a function only of the action variables $\mathbf{n}$. This follows from the fact that the formal Fourier series introduced to represent the solution of the classical problem defines a canonical transformation to the correct action angle variables (see below).

For the quantity just evaluated, as well as for the potential energy,

$$
\lim \langle\mathbf{n}|V| \mathbf{n}\rangle=\langle V\rangle,
$$

the relative error is of order $\left(1 / n^{2}\right)$, provided that we use the definition (3.1).

If we combine Eqs. (4.2) and (4.3) we have

$$
\lim \langle\mathbf{n}|L| \mathbf{n}\rangle=\langle L\rangle \text {. }
$$

Since this expression is the semiclassical limit of a quantity stationary with respect to variations of matrix elements of the coordinates, keeping energy differences fixed, we expect (4.4) to be stationary with respect to variations of the associated Fourier components, keeping the frequency components fixed. Conversely, requiring the stationary property

$$
\frac{\delta\langle L\rangle}{\delta x_{i,-\mathbf{k}}}=0
$$

keeping the frequencies fixed, yields Lagrange's equations in Fourier component form, namely,

$$
(\mathbf{k} \cdot \boldsymbol{\omega})^{2} x_{i, \mathbf{k}}(\mathbf{n})=\frac{\partial\langle V\rangle}{\partial x_{i,-\mathbf{k}}} .
$$

Considered from the purely classical point of view, the solution of Eq. (4.6) may be undertaken from several standpoints. The choice of real Fourier coefficients, adhered to throughout the present development, has already determined half of the initial conditions, namely, all components of the velocity vanish initially. If we then specify $N$ fundamental Fourier components of the coordinates, we thereby define a scheme, provided that a solution exists, that determines the remaining Fourier components as well as the $N$ frequencies. An alternative scheme, more integral to the variational principle, is to specify $N$ (incommensurable) frequencies and calculate the Fourier amplitudes from the equations of motion. In this paper, however, our interest will be in adjoining $N$ quantization conditions to the equations of motion. The resulting set, if it has a solution, must then determine the Fourier amplitudes and the frequencies.

We apply the EBK quantization condition (restoring $\hbar$ for the instant)

$$
I_{i}=\left(n_{i}+\frac{1}{4} \alpha_{i}\right) \hbar
$$

where $\alpha_{i}$, the Maslov index, has the value 2 for an uncoupled vibrational degree of freedom and for the models studied in Sec. VII. (For a more general discussion of the significance of this index and how it is determined in a given case, see Ref. [5].) The action $I_{i}$ is given by the equivalent expressions $[13,17,21]$

$$
I_{i} \equiv \frac{\delta\langle L\rangle}{\delta \omega_{i}}=\sum_{\mathbf{k}} k_{i}(\mathbf{k} \cdot \boldsymbol{\omega}) \mathbf{x}_{\mathbf{k}} \mathbf{x}_{-\mathbf{k}}=\left\langle\mathbf{p} \cdot \frac{\partial}{\partial \theta_{i}} \mathbf{x}\right\rangle,
$$


where, in the last form, $\mathbf{p}$ is the momentum vector of the system. This completes the formal statement of the semiclassical quantization for invariant tori.

\section{B. Connection between EBK quantization and commutation relations}

We have, in fact, given two quantization schemes in this paper: the completely quantum one consisting of Heisenberg's equations of motion adjoined to diagonal elements of the canonical commutation relations and the semiclassical one consisting of the classical equations of motion in Fourier component form adjoined to EBK quantization conditions. But a part of the logical story is clearly missing. On the one hand, we have derived the classical equations of motion from the quantum ones using correspondence principle arguments modified only minimally from historical forms. On the other hand, although EBK quantization can also be derived, up to the value of the Maslov index, from the correspondence principle, what has not been made clear is the relationship between the canonical commutation relations and the EBK quantization conditions. Though it is hard to believe that this question has not been addressed at some point in the history of quantum mechanics, we have never encountered such a discussion in the literature. Therefore, for the sake of completeness, we include it at this juncture.

Consider first the diagonal matrix element of the commutation relation

$$
\begin{aligned}
\lim \left\langle\mathbf{n}\left|\left[x_{i},\left[H, x_{i}\right]\right]\right| \mathbf{n}\right\rangle & =-i \lim \left\langle\mathbf{n}\left|\left[x_{i}, p_{i}\right]\right| \mathbf{n}\right\rangle \\
& =\left\langle\sum_{j}\left[\frac{\partial x_{i}}{\partial \theta_{j}} \frac{\partial p_{i}}{\partial n_{j}}-\frac{\partial x_{i}}{\partial n_{j}} \frac{\partial p_{i}}{\partial \theta_{j}}\right]\right\rangle \\
& =\sum_{j, \mathbf{k}} \frac{\partial}{\partial n_{j}} k_{j}(\mathbf{k} \cdot \boldsymbol{\omega}) x_{i, \mathbf{k}} x_{i,-\mathbf{k}} .
\end{aligned}
$$

The basic result exhibited here, that follows from Lemma 3, is that the limit of the diagonal element of the commutator is the time average of the corresponding Poisson bracket (PB). On the other hand, we observe that the time average of the fundamental Lagrange bracket $\left\{\theta_{i}, n_{i}\right\}$ has the value

$$
\begin{aligned}
\left\langle\left\{\theta_{i}, n_{i}\right\}\right\rangle & \equiv\left\langle\sum_{j}\left[\frac{\partial x_{j}}{\partial \theta_{i}} \frac{\partial p_{j}}{\partial n_{i}}-\frac{\partial x_{j}}{\partial n_{i}} \frac{\partial p_{j}}{\partial \theta_{i}}\right]\right\rangle \\
& =\frac{\partial}{\partial n_{i}} \sum_{j, \mathbf{k}} k_{i}(\mathbf{k} \cdot \boldsymbol{\omega}) x_{j, \mathbf{k}} x_{j,-\mathbf{k}} \\
& =\frac{\partial}{\partial n_{i}} I_{i}=1 .
\end{aligned}
$$

Thus the EBK quantization condition is related to the Lagrange bracket (LB) rather than to the PB.

It is, of course, well known [41] that one set of fundamental brackets implies the other. But for this purpose we must consider the full brackets rather than just their time averages over the torus. We illustrate the argument for one degree of freedom. We thus wish to show that the formal Fourier series

$$
x(n, \theta)=\sum_{q} x_{q}(n) \exp (i q \theta)
$$

$$
p(n, \theta)=\sum_{q} i q \omega x_{q}(n) \exp (i q \theta)
$$

satisfy the PB condition

$$
[x, p]_{\mathrm{PB}}=[x, p]_{\theta, n}=1 .
$$

provided, of course, that Eqs. (4.11) satisfy the equations of motion. In that case the Fourier series represent the canonical transformation between the given canonical coordinates $x, p$ and the exact angle-action variables $\theta, n$. By working out the Fourier series for the $\mathrm{PB}$, requiring that the constant term be unity and that the other Fourier components vanish, we find, with the help of a linear transformation of the summation indices, that

$$
\begin{aligned}
\delta_{q, 0}= & \sum_{k}\left[\left(k^{2}-\frac{1}{4} q^{2}\right) x_{k+(1 / 2) q} \frac{\partial}{\partial n}\left(\omega x_{-k+(1 / 2) q}\right)\right. \\
& \left.+\left(k-\frac{1}{2} q\right)^{2} \omega x_{-k+(1 / 2) q} \frac{\partial}{\partial n} x_{k+(1 / 2) q}\right]
\end{aligned}
$$

In the sum, $k$ takes on both integral and half integral values. For $q=0$ this expression reduces to the one-dimensional form of (4.9). The vanishing of the remaining Fourier components must be a consequence, we suspect, of the vanishing of the off diagonal elements of the commutator, i.e., it must be the classical limit of this property. This can be verified directly, using Lemma 3(a). As we emphasized at the beginning of this discussion, the equations of motion must be involved, as indeed they are, in the vanishing of these offdiagonal elements.

Using the results derived in Sec. II B, we can extend the detailed considerations just given to the multidimensional problem. This is in fact the interesting case, since in the one-dimensional case the PB and LB are indistinguishable, a fact that will be used later to derive the $\mathrm{WKB}$ quantization condition from the commutation relation. The correct value of the Maslov indices must somehow also be implied in the multidimensional derivation of the EBK quantization conditions. The only derivation we can supply at the moment is one based on starting with a separable system and assuming adiabatic invariance as one turns up the coupling.

It may be of some interest to derive the $q \neq 0$ part of (4.13) directly from the classical variational principle. For this purpose, we find it necessary to retain the full structure of the quantum variational principle, so that in the classical limit we have not only an average over a given torus, but, in addition, from the trace operation, an integral over all tori. In other words, we consider an average over phase space and indicate it by a double bracket notation, e.g.,

$$
\langle\langle L\rangle\rangle \equiv \int d n d \theta L(\theta, n),
$$

where the phase average of $L$ has the form 
TABLE I. Concepts of the theory of invariant tori as the classical limit of the concepts associated with Heisenberg matrix mechanics in the energy-diagonal representation.

\begin{tabular}{|c|c|c|}
\hline & Quantum & Semiclassical \\
\hline 1 & $\begin{array}{l}\text { Matrix elements of } x, p \\
\qquad\langle n|x| m\rangle,\langle n|p| m\rangle\end{array}$ & $\begin{array}{l}\text { Fourier components of } x(I, \theta), p(I, \theta) \\
\qquad x_{k}(I), p_{k}(I)\end{array}$ \\
\hline 2 & Trace variational principle & Constrained variational principle \\
\hline & $\delta \operatorname{Tr}(H-i p[H, x])=0$ & $\delta\left(\left(H-\omega p \frac{\partial x}{\partial \theta}\right)\right)=0$ \\
\hline 3 & $\begin{array}{l}\text { Matrix element of equation of motion } \\
\qquad\left(E_{n+k}-E_{n}\right)^{2} x_{n+k, n}=\left(\frac{d V}{d x}\right)_{n+k, k}\end{array}$ & $\begin{array}{l}\text { Fourier component of equation of motion } \\
\qquad(k \omega)^{2} x_{k}=\frac{d\langle V\rangle}{d x_{-k}}\end{array}$ \\
\hline 4 & $\begin{array}{l}\text { Commutation relations } \\
\qquad\langle n|[x, p]| n\rangle=i\end{array}$ & $\begin{array}{l}\text { Quantization of the action } \\
\qquad I=\left\langle p \frac{\partial x}{\partial \theta}\right\rangle=n+ \\
\frac{1}{2}\end{array}$ \\
\hline 5 & $\begin{array}{l}\text { Energy is the diagonal element of } H \\
\qquad E_{n}=\langle n|H| n\rangle\end{array}$ & $\begin{array}{l}\text { Energy is the phase average of } H \\
\qquad E(I)=\langle H\rangle\end{array}$ \\
\hline 6 & $\begin{array}{l}\text { Quantum frequency } \\
\omega_{n+k, n} \equiv E_{n+k}-E_{n}\end{array}$ & $\begin{array}{l}\text { Classical frequency } \\
\qquad \omega=d E(I) / d I\end{array}$ \\
\hline 7 & $\begin{array}{l}\text { Operator } \\
A(x, p)\end{array}$ & $\begin{array}{c}\text { Dynamical variable } \\
A(x(I, \theta), p(I, \theta))\end{array}$ \\
\hline 8 & $\begin{array}{l}\text { Matrix elements } \\
\qquad\langle n|A| n+k\rangle\end{array}$ & $\begin{array}{c}\text { Fourier coefficients } \\
A_{k}(I)=\int \frac{d \theta}{2 \pi} A(I, \theta) \exp (-i k \theta)\end{array}$ \\
\hline 9 & $\begin{array}{c}\text { Energy is diagonal } \\
\sum_{k \neq 0}\langle n|H| n+k\rangle\langle n+k|H| n\rangle=0\end{array}$ & $\begin{array}{l}\text { Hamiltonian independent of angles } \\
\qquad \sum_{k \neq 0} H_{k} H_{-k}=0\end{array}$ \\
\hline
\end{tabular}

$$
\langle L\rangle=\frac{1}{2} i^{2}\left\langle\left(\frac{\partial x}{\partial \theta}\right)^{2}\left(\frac{\partial H}{\partial n}\right)^{2}\right\rangle-\langle V\rangle
$$

We now subject the system to an arbitrary infinitesimal canonical transformation about the exact solution. As a consequence of the equations of motion, the only nonvanishing contribution comes from the first term of (4.15) and has the form

$$
\begin{aligned}
\delta\langle\langle L\rangle\rangle & =-\left\langle\left\langle\left(\frac{\partial x}{\partial \theta}\right)^{2} \frac{\partial H}{\partial n} \delta \frac{\partial H}{\partial n}\right\rangle\right) \\
& =\left\langle\left\langle\delta H\left[\frac{\partial^{2} H}{\partial n^{2}}\left(\frac{\partial x}{\partial \theta}\right)^{2}+\frac{\partial H}{\partial n} \frac{\partial}{\partial n}\left(\frac{\partial x}{\partial \theta}\right)^{2}\right]\right\rangle\right\rangle .
\end{aligned}
$$

Here the second line has been obtained by an integration by parts with respect to $n$ with boundary terms dropped. This requirement is clearly the classical analog of the invariance of the trace, as is the requirement that (4.16) vanish. Finally, if we assume that $\delta H$ is expanded in a Fourier series with no constant term and otherwise arbitrary Fourier coefficients, we are forced to the conclusion that all Fourier components of the expression in square brackets, other than the constant term, vanish. It is in fact easy to see that this conclusion coincides with the corresponding statement contained in (4.13).
It is, finally, important to refer to the classical limit of the property that the quantum theory is formulated in the representation in which $H$ is diagonal. The limit of this property is precisely the condition that when the canonical transformation to the exact action-angle variables has been carried out, it is signaled by the vanishing of the Fourier series for the classical $H$, other than the constant term. In applications, we shall utilize this requirement in both its quantum and classical aspects as a test of the convergence of our solutions. For example, in the classical limit, the difference

$$
\left\langle H^{2}\right\rangle-\langle H\rangle^{2}=\sum_{k \neq 0} H_{k} H_{-k}
$$

should vanish.

We summarize in Table I the analogy that has now been fully established between Heisenberg matrix mechanics in the energy-diagonal representation and the theory of invariant tori. For the sake of simplicity, the notation appropriate to one degree of freedom has been utilized. All entries have been described in the preceding text. Note that we have included the Hamiltonian rather than the Lagrangian form of the variational principle.

\section{FURTHER STUDY OF THE COMMUTATION RELATIONS}

\section{A. One dimension and the WKB quantization rule}

In the following lines we present a derivation of the WKB quantization rule for bound states that is simpler than the one 
that we have previously published [20]. A diagonal element in a state $n$ of the canonical commutation relation may be written (using reality conditions)

$$
\sum_{n^{\prime}=0}^{\infty} 2 x_{n n^{\prime}} i p_{n n^{\prime}}=1
$$

Change $n \rightarrow n^{\prime \prime}$ and sum this equation from 0 to $n$. Divide the resulting double sum into two terms. The first, defined by taking both sums from 0 to $n$, vanishes by antisymmetry (the matrix elements of $x$ are symmetric under exchange of indices, those of $p$ antisymmetric). We are left with the sum

$$
\sum_{n^{\prime \prime}=0}^{n} \sum_{n^{\prime}=n+1}^{\infty} 2 x_{n^{\prime \prime} n^{\prime}} i p_{n^{\prime \prime} n^{\prime}}=n+1 \text {. }
$$

With our standard definition

$$
x_{n^{\prime}-n}\left[\frac{1}{2}\left(n+n^{\prime}\right)\right] \equiv x_{n n^{\prime}}
$$

and a change of indices

$$
n^{\prime \prime}=n-\nu, \quad n^{\prime}=n+k-\nu,
$$

(5.2) becomes

$2 \sum_{k=1}^{\infty} \sum_{\nu=0}^{k-1} x_{k}\left(n+\frac{1}{2} k-\nu\right) i p_{k}\left(n+\frac{1}{2} k-\nu\right)=n+1$.

Equation (5.5) is still exact and can be used in quantum calculations. Expanding about the semiclassical value $x_{k}(n)$ and keeping terms that contribute at most to order $n$ and to order unity, we find

$$
\sum_{k=1}^{\infty}\left[2 k x_{k}(n) i p_{k}(n)+\frac{d}{d n}\left[k x_{k}(n) i p_{k}(n)\right]+\cdots\right]=n+1 .
$$

(To obtain the form of the second term on the left-hand side of this equation requires a careful examination and grouping of terms that contribute.) The way to read this equation is to understand that the first term contains contributions of order $n$ and smaller, whereas the second term is at most of order unity and at the same time is half the derivative with respect to $n$ of the first term. It follows that this second term has the value $\frac{1}{2}$. We thus derive the one-dimensional Cartesian WKB quantization rule

$$
2 \sum_{k=1}^{\infty} k x_{k}(n) i p_{k}(n)=n+\frac{1}{2} .
$$

A similar tour de force does not work in two or more dimensions. We omit the uninspiring details. The moral of the story is that we are "lucky" to have the EBK quantization rules for semiclassical quantization. Our aim when we started the investigation of this section was to discover an alternative semiclassical scheme based directly on the commutation relations. The conclusion, which we believe to be firm, is that this is not possible except in the one-dimensional case.

\section{B. Remarks on quantum aspects}

Consider a two-dimensional system and suppose it to possess only bound states. (In any event we use a notation with discrete labels only.) We can write a Heisenberg scheme that should be valid whether we deal with the "regular" spectrum or the "irregular" one [17]. We simply order the energy levels of a two-dimensional system with coordinates $x, y$, in a linear sequence, $N=0,1, \ldots$. A calculation can be based on the equations

$$
\begin{gathered}
\left(E_{N^{\prime}}-E_{N}\right)^{2} x_{N N^{\prime}}=-\left(F_{x}\right)_{N N^{\prime}}, \\
\left(E_{N^{\prime}}-E_{N}\right)^{2} y_{N N^{\prime}}=-\left(F_{y}\right)_{N N^{\prime}}, \\
\sum_{N^{\prime}}\left(E_{N^{\prime}}-E_{N}\right)\left|x_{N N^{\prime}}\right|^{2}=1,
\end{gathered}
$$

$$
\sum_{N^{\prime}}\left(E_{N^{\prime}}-E_{N}\right)\left|y_{N N^{\prime}}\right|^{2}=1,
$$

where $E_{N}$ are the exact eigenvalues. These equations can be viewed in two ways, either as a set of sum rules to be satisfied by the exact solutions of the quantum mechanical problem, found by some other means such as matrix diagonalization in a basis, or else as the foundation for a computational scheme involving the solution of nonlinear equations. For a well-defined quantum system (Hamiltonian bounded from below), we shall certainly be able to do a diagonalization and subsequently check the above equations. Since this should be possible both for the regular and for the irregular spectrum, this raises the hope that some version of the quantum matrix method can also be applied to the chaotic regime.

Toward this end, we note that by summing the commutation relations from 0 to $N$, we can replace (5.9) by the positive sums (this involves the same argument as in the onedimensional case)

$$
\begin{aligned}
& \sum_{N^{\prime}=N+1}^{\infty} \sum_{N^{\prime \prime}=0}^{N}\left(E_{N^{\prime}}-E_{N^{\prime \prime}}\right)\left|x_{N^{\prime \prime} N^{\prime}}\right|^{2}=N+1, \\
& \sum_{N^{\prime}=N+1}^{\infty} \sum_{N^{\prime \prime}=0}^{N}\left(E_{N^{\prime}}-E_{N^{\prime \prime}}\right)\left|y_{N^{\prime \prime} N^{\prime}}\right|^{2}=N+1 .
\end{aligned}
$$

These relations are interesting because they guarantee the convergence of certain sums and thus imply that the matrix elements cannot spread out too far as a function of energy differences.

\section{APPLICATION OF MATRIX MECHANICS AND COMPARISON WITH THE SEMICLASSICAL APPROXIMATION}

In this section we present illustrative applications of the matrix mechanics method for several simple models and compare the results with those of the semiclassical approximation as well as with the results of exact diagonalization. With a given semiclassical approximation, as defined below, we shall associate the matrix method, which will be described as a sequence of approximations that should approach more and more closely to the exact result. Therefore 
we may anticipate that at a sufficiently high order of approximation its accuracy will exceed that of the semiclassical result. It is not, however, guaranteed to do this automatically for the simple reason that the quantum method that we shall describe does not have a true semiclassical limit, but is specifically designed to be most accurate for small quantum numbers. For this reason, we shall also describe an alternative method that starts with the EBK theory and specifically calculates quantum corrections to it. Numerical results for all three methods are presented at the end of this section.

Imagine that we have a two-dimensional system described by a Hamiltonian

$$
\begin{aligned}
H & =\frac{p_{1}^{2}}{2}+\frac{p_{2}^{2}}{2}+\frac{1}{2} \lambda_{1}\left(x^{1}\right)^{2}+\frac{1}{2} \lambda_{2}\left(x^{2}\right)^{2}+V^{\text {anharmonic }}\left(x^{1}, x^{2}\right) \\
& \equiv \frac{\mathbf{p}^{2}}{2}+\frac{|\lambda \cdot \mathbf{x}|^{2}}{2}+V^{\text {anharmonic }}(\mathbf{x})
\end{aligned}
$$

However, the methods described here are more general and can be used with a system with many degrees of freedom. We shall describe these methods, in general, by referring to an $n$-dimensional system and give examples and show results for the specific cases $n=1$ and $n=2$. For improved clarity, we have shifted the coordinate index from subscript to superscript.

For the solution of the algebraic formulation of the semiclassical approximation we utilize an iterative method described by Percival and Pomphrey $[15,16]$. For the corresponding fully quantum calculations, we shall describe a quantum extension of this method. We have also carried out calculations using the Newton-Raphson method, but these will not be discussed here.

\section{A. Semiclassical iterative method}

Following Percival and Pomphrey, this method utilizes the semiclassical equations of motion, given for Fourier components $\mathbf{k}$ (in terms of the above Hamiltonian),

$$
\left[\lambda-(\mathbf{k} \cdot \omega)^{2}\right] \mathbf{x}_{\mathbf{k}}=\mathbf{F}_{\mathbf{k}}(\mathbf{x}),
$$

where

$$
\mathbf{F}(\mathbf{x})=-\nabla V^{\text {anharmonic }}(\mathbf{x}),
$$

and $\omega=\left(\omega_{1}, \omega_{2}, \ldots, \omega_{n}\right)$ is the vector of frequencies of the system. When there is no anharmonic driving force, $\omega_{i}^{2}=\lambda_{i}$. The vector $\mathbf{k}$ is an $n$-dimensional sequence of integers, positive and negative: $\mathbf{k}=\left(k_{1}, k_{2}, \ldots, k_{n}\right)$. It is also useful, here, to define the vectors $\mathbf{1}=(1,0,0, \ldots, 0)$, $\mathbf{2}=(0,1,0, \ldots, 0), \mathbf{i}=(0, \ldots, 1, \ldots, 0)$, where the 1 is in the $i$ th place and all other indices are zero. Thus $\mathbf{k} \cdot \mathbf{i}=k_{i}$. In order to solve the equations that describe the semiclassical limit in algebraic terms, we must truncate the Fourier series by specifying a maximum integer vector $|\mathbf{K}|$. The meaning of the absolute value is that we define this limit symmetrically with respect to the origin in $\mathbf{k}$ space. We set $x_{\mathbf{k}}^{i}=0$ for $|\mathbf{k}|>|\mathbf{K}|$.

The procedure begins from the harmonic limit, where the only nonzero Fourier components are $x_{ \pm \mathbf{i}}^{i}$ As an example, for a two-dimensional system, where we write $x^{1}=x$ and $x^{2}=y$, the only nonzero components, initially, are $x_{ \pm 1,0}$ and $y_{0, \pm 1}$. All others vanish. We shall refer to these as the driving components. The values of these initial nonzero components are computed from the action conditions Eq. (4.8), namely, $x_{ \pm \mathbf{i}}^{i}=\sqrt{I_{i} / 2 \omega_{i}}$, where $I_{i}$ is the action of the $i$ th degree of freedom, as given by Eq. (4.7).

With these initial values, we can then calculate initial values for the Fourier components of the nonharmonic part of the force. Improvements to the frequencies $\omega_{i}$ are then obtained from the equations of motion for the driving components,

$$
\omega_{i}^{2}=\lambda_{i}-\frac{F_{\mathbf{i}}^{i}}{x_{\mathbf{i}}^{i}} .
$$

With these new and improved frequencies, other Fourier components can be obtained from the remaining equations of motion. In particular, we compute

$$
x_{\mathbf{k}}^{i}=\frac{F_{\mathbf{k}}^{i}(\mathbf{x})}{\lambda^{i}-(\mathbf{k} \cdot \boldsymbol{\omega})^{2}} \quad \text { for } \quad \mathbf{k} \neq \mathbf{i}
$$

This completes one cycle of iteration. We now return to the EBK quantum conditions, and using the calculated values of the frequencies and of the nondriving Fourier components we compute new and improved values for the driving components. Explicitly,

$$
x_{\mathbf{i}}^{i}=\sqrt{\frac{1}{2 \omega_{i}}\left[I_{i}-\sum_{\mathbf{k} \neq \mathbf{i}} k_{i}(\mathbf{k} \cdot \boldsymbol{\omega}) \mathbf{x}_{\mathbf{k}} \mathbf{x}_{-\mathbf{k}}\right]} .
$$

For the special case $n=2$, we have

$x_{ \pm 1,0}$

$$
=\sqrt{\frac{I_{x}-\sum_{\left(k_{1}, k_{2}\right) \neq( \pm 1,0)} k_{1}\left(x_{k_{1}, k_{2}}^{2}+y_{k_{1}, k_{2}}^{2}\right)\left(k_{1} \omega_{1}+k_{2} \omega_{2}\right)}{2 \omega_{1}}}
$$

$y_{0, \pm 1}$

$$
=\sqrt{\frac{I_{y}-\sum_{\left(k_{1}, k_{2}\right) \neq(0, \pm 1)} k_{2}\left(x_{k_{1}, k_{2}}^{2}+y_{k_{1}, k_{2}}^{2}\right)\left(k_{1} \omega_{2}+k_{2} \omega_{2}\right)}{2 \omega_{2}}} .
$$

Once more we calculate the improved values of the frequencies and then of the remaining components and continue to loop until the energy converges.

\section{B. Quantum iterative method}

In this section we discuss the modifications to the semiclassical perturbative method that are necessary in order to have a completely quantum calculation. The major complication of the quantum approach is that in our version of the 
Heisenberg method, we must consider the coupling of eigenstates both in the equations of motion and in the commutation relations. To deal with this problem in a practical way will necessitate the introduction of two cutoff parameters, compared to the one used in the semiclassical case.

To explain this difference within the simplest context, we return to a one-dimensional notation and recall how the semiclassical approximation is defined. In the quantum theory, together with the matrix element

$$
\left\langle n-\frac{1}{2} k|x| n+k\right\rangle \equiv x_{k}(n),
$$

which is the focus of a given semiclassical calculation, we will also encounter, for a fixed value of $k$, matrix elements such as

$$
x_{k}(n+b)=x_{k}(n)+b \frac{d x_{k}(n)}{d n}+\cdots .
$$

In the quantum theory, we treat on an equal footing a certain range of values of $b$, symmetrically disposed with respect to $b=0$, and refer to the maximum value of $b$ thus included as the bandwidth $B$. Normally it is necessary to include still larger values of $b$ in our equations in order to be consistent numerically. For these we normally make the closure approximation [cf. (6.10)]

$$
x_{k}(n+B+a) \cong x_{k}(n+B),
$$

valid for small $a$. The introduction of a closure approximation is an invariable aspect of all approximate calculations made by the form of matrix mechanics described in this work; it plays the role of reducing the number of unknowns to the number of equations utilized. Finally, the significance of the parameter $K$, the maximum Fourier component, is the same for the quantum as for the classical calculation. Similar ideas apply to calculations that work from the ground state up (and therefore do not have a semiclassical limit), except that the values of the "Fourier" index $k$ and of the parameter $b$ take on only positive values, as we shall now see.

Consider a typical matrix element $\langle\mathbf{m}|O| \mathbf{n}\rangle$ of an operator $O$ (in general a coordinate). If we have chosen an ordering of the integer vectors, we can suppose that $\mathbf{n}>\mathbf{m}$ is defined and $\mathbf{k} \equiv \mathbf{n}-\mathbf{m}$ is the analog of a Fourier component. Since the first state always considered in the quantum method to be described is the ground state $\mathbf{m}=\mathbf{0}, \mathbf{K}$ coincides with the maximum value of $\mathbf{n}$ that we include in the calculation. However, this specification does not completely define the calculation. The crudest way to define a closed scheme for fixed $\mathbf{K}$ is to allow only matrix elements with $\mathbf{m}=\mathbf{0}$. But the equations of motion for the matrix elements $\langle\mathbf{0}|O| \mathbf{n}\rangle$ will necessarily bring in matrix elements with $\mathbf{m} \neq \mathbf{0}$. In order to have a closed scheme involving only the quantities specified, we must adjoin a closure approximation that relates the unwanted matrix elements to those that belong to the allowed set. The crudest such approximation is to relate matrix elements by an equal displacement of both sets of quantum numbers, in analogy with (6.11),

$$
\langle\mathbf{m}|O| \mathbf{n}\rangle \cong\langle\mathbf{0}|O| \mathbf{n}-\mathbf{m}\rangle .
$$

(However, a better approximation at this point is to use harmonic-oscillator results for the large matrix elements that are nonvanishing in the uncoupled limit.) Equation (6.12) defines the zero bandwidth, $B=0$ approximation in the present case.

To improve the scheme, we increase the bandwidth $B$. Thus, in a $B=1$ approximation, in analogy with the onedimensional case, we treat without closure those values of m that comprise both the ground state and those states that are coupled to the ground state by harmonic-oscillator matrix elements. By suitable extension we can define a scheme for arbitrary $B$, with the understanding that the maximum value of $\mathbf{m}$, called $\mathbf{M}$, cannot exceed $\mathbf{K}$. A closure approximation, generalizing (6.12), needs to be applied now only to relate matrix elements outside the expanded scheme to those in which the left-hand state is $\mathbf{M}$. As $B$ increases, we find that the values obtained for low-lying states become more and more accurate, as well as insensitive to the closure approximation. The values of $B$ and of $\mathbf{K}$ determine the number of equations of motion and commutation relations that have to be used to evaluate the set of included matrix elements and energy differences. This number is a small multiple of $B$ times the number of equations for the semiclassical approximation associated with the same value of $\mathbf{K}$.

Turning to the detailed scheme, we begin from the harmonic limit. In this limit, all the energies are equally spaced. Thus, if we define quantum frequencies to be the energy differences

$$
\langle\mathbf{n}|H| \mathbf{n}\rangle-\langle\mathbf{m}|H| \mathbf{m}\rangle=E_{\mathbf{n}}-E_{\mathbf{m}} \equiv \omega_{\mathbf{n}, \mathbf{m}},
$$

then the harmonic limit is defined by

$$
\omega_{\mathbf{n} \pm \mathbf{i}, \mathbf{n}}= \pm \sqrt{\lambda_{i}} .
$$

Here the vector $\mathbf{i}$ is the same as was introduced above, namely, it is an $n$-dimensional vector whose entries are all zero except for the $i$ th one, whose value is unity.

From the well-known solution for the harmonic oscillator, we also know the driving matrix elements $x_{\mathbf{n}+\mathbf{i}, \mathbf{n}}^{i}$ It is integral to our method that this result need not be put in from the outside but follows by application of the canonical commutation relations, given the result expressed by (6.14). Actually, the full statement is that for the harmonic case, as is well known, the simultaneous solution of the equations of motion and of the commutation relations yields the starting values of the quantum frequencies and of the driving matrix elements. In turn, we can calculate the lowest approximation to the force $F_{\mathbf{n}, \mathbf{n + i}}^{i}$. This completes a single cycle of the quantum iteration procedure.

Next, the quantum frequencies are improved by using the generalization of Eq. (6.4),

$$
\omega_{\mathbf{n}+\mathbf{i}, \mathbf{n}}^{2}=\lambda_{i}-\frac{F_{\mathbf{n}, \mathbf{n}+\mathbf{i}}^{i}}{x_{\mathbf{n}, \mathbf{n}+\mathbf{i}}^{i}}
$$

For further work, we write

$$
\begin{gathered}
\omega_{\mathbf{n}, \mathbf{m}}=\omega_{\mathbf{m}+\mathbf{a}, \mathbf{m}}, \\
\mathbf{a}=a_{1} \mathbf{1}+a_{2} \mathbf{2}+\cdots+a_{n} \mathbf{n .}
\end{gathered}
$$


It is straightforward to write this energy difference as a sum of fundamental energy differences or quantum frequencies in which just one of the quantum numbers changes by a unit. This is important in counting that the number of variables in the problem is determined by the equations of motion and a set of diagonal elements of the commutation relations. With the new and improved energy differences, it is possible to compute the perturbative quantum matrix elements from the equations of motion

$$
x_{\mathbf{n}, \mathbf{n}+\mathbf{a}}^{i}=\frac{F_{\mathbf{n}, \mathbf{n}+\mathbf{a}}^{i}}{\omega_{\mathbf{n}+\mathbf{a}, \mathbf{n}}^{2}} \text { for } \mathbf{a} \neq \mathbf{i} .
$$

Finally, we construct new "fundamental" matrix elements from the diagonal matrix elements of the canonical commutation relations. Toward this end, we solve the $n$ equations $(i=1, \ldots, n)$

$$
\sum_{\mathbf{m}}\left(x^{i}\right)_{\mathbf{j}, \mathbf{m}}^{2} \omega_{\mathbf{m}, \mathbf{j}}=\frac{1}{2}
$$

for $x_{\mathbf{m}+\mathbf{i}, \mathbf{m}}^{i}$ These equations replace the quantum conditions for the action variables, used in the semiclassical approximation, and, as already stated, play the same role as the latter did in providing additional equations needed to have a determined set. We have thus identified the three distinct elements that are used in an iteration cycle: the commutation relations, the equations of motion for the "driving" matrix elements, and the equations of motion for the "small" matrix elements.

\section{Quantum corrections to the semiclassical approximation}

We start by reminding the reader that the semiclassical approximation to the energy $E(n)=\langle n|H| n\rangle$ (in a onedimensional notation, is obtained by expanding energy differences and quantum matrix elements about certain values that are identified as semiclassical ones and dropping higherorder terms. With the definitions

$$
\begin{gathered}
\omega(n)=E\left(n+\frac{1}{2}\right)-E\left(n-\frac{1}{2}\right), \\
k=n^{\prime \prime}-n^{\prime}, \\
\bar{n}=\frac{1}{2}\left(n^{\prime}+n^{\prime \prime}\right),
\end{gathered}
$$

we have, to second order,

$$
\begin{aligned}
&\left\langle n^{\prime}|x| n^{\prime \prime}\right\rangle=x_{k}(n)+(\bar{n}-n) \frac{d x_{k}(n)}{d n}+\frac{1}{2}(\bar{n}-n)^{2} \frac{d^{2} x_{k}(n)}{d^{2} n}, \\
& E(n+k)-E(n)= k \omega(n)+\frac{1}{2} k^{2} \frac{d \omega(n)}{d n} \\
&+\left(\frac{1}{6} k^{3}-\frac{1}{24} k\right) \frac{d^{2} \omega(n)}{d^{2} n},
\end{aligned}
$$

where the derivation of the second of these equations requires attention to the definition (6.20).

To obtain quantum corrections to the energy, we must retain and compute the values of the first and second deriva- tive terms in (6.23) and (6.24). These equations are expansions in $(1 / n)$ and we need terms up to second order because, as we have shown, the first-order terms do not contribute to the energy.

The calculation is straightforward. Reverting to an $n$-dimensional notation, we recall that the semiclassical quantization program is based on the equations

$$
(\mathbf{k} \cdot \boldsymbol{\omega})^{2} x_{k}^{i}=F_{k}^{i},
$$

$$
\sum_{j, \mathbf{k}} k_{i}(\mathbf{k} \cdot \boldsymbol{\omega}) x_{k}^{j} x_{-k}^{j}=\left(n_{i}+\frac{1}{4} \alpha_{i}\right),
$$

where $F_{k}^{i}$ is a Fourier component of the $i$ th component of the force and $\alpha_{i}$ is a Maslov index. Let us write these equations succinctly as

$$
\Psi_{a}(z)=0
$$

where $a$ is a label that enumerates in turn the equation set (6.25) and (6.26), putting them in a one to one correspondence with the Fourier components and with the frequencies. Correspondingly, $z=\left\{z_{a}\right\}=\left\{x_{k}^{i}, \omega_{i}\right\}$.

We now treat these equations as if they are true for a continuous range of $n$ and compute the first and second derivatives, leading to the equations,

$$
\begin{gathered}
\frac{\partial \Psi_{a}}{\partial z_{b}} \frac{\partial z_{b}}{\partial n_{i}}=s_{a i}, \\
\frac{\partial \Psi_{a}}{\partial z_{b}} \frac{\partial^{2} z_{b}}{\partial n_{i} \partial n_{j}}=-\frac{\partial^{2} \Psi_{a}}{\partial z_{b} \partial z_{c}} \frac{\partial z_{b}}{\partial n_{i}} \frac{\partial z_{c}}{\partial n_{j}} .
\end{gathered}
$$

Here the column matrix $s_{a i}$, consisting of all zeros and a single value of unity, has its origin in the right-hand side of Eq. (6.26). Both of the above sets of equations are linear and inhomogeneous and will have a solution provided that the matrix

$$
S_{a, b}=\frac{\partial \Psi_{a}}{\partial z_{b}}
$$

is nonsingular. (See Sec. VII for further discussion of the uses of this matrix.) The solutions of these equations provides us with the necessary input for calculating the leading quantum corrections to the energy.

\section{Numerical illustrations}

We consider first the one-dimensional inverted quartic potential

$$
H=\frac{1}{2}\left(p^{2}+x^{2}\right)+b x^{4}
$$

with $b<0$. For this model, as well as for the twodimensional model considered below, it is understood that these potentials do not define quantum-mechanical systems. Nevertheless, diagonalization in a large, but not too large, basis will yield eigenvalues that we can treat as those of a bound system. Correspondingly, the approximation methods described above will also yield values that we can take seriously and compare with the "exact" results. In the following 
TABLE II. Representative values of the energy for states $n$ of a one-dimensional quartic oscillator, for several values of the anharmonicity parameter $b . E_{i \text { diag }}$ denotes the energy calculated by direct diagonalization, $E_{\mathrm{sc}}$ is the semiclassical approximation, $E_{\mathrm{sc}}+\Delta E$ includes the leading quantum correction to the semiclassical result, and $E_{q}$ is the quantum result obtained by the Heisenberg method.

\begin{tabular}{ccrrrr}
\hline \hline$b$ & $n$ & \multicolumn{1}{c}{$E_{\text {diag }}$} & \multicolumn{1}{c}{$E_{\mathrm{sc}}$} & \multicolumn{1}{c}{$E_{\mathrm{sc}}+\Delta E$} & \multicolumn{1}{c}{$E_{q}$} \\
\hline-0.002 & 0 & 0.498489 & 0.499248 & 0.498500 & 0.498489 \\
& 5 & 5.405378 & 5.406235 & 5.405521 & 5.405377 \\
& 10 & 10.145887 & 10.146877 & 10.146244 & 10.145887 \\
-0.005 & 0 & 0.496182 & 0.498112 & 0.496249 & 0.496182 \\
& 5 & 5.249313 & 5.252083 & 5.250752 & 5.249314 \\
& 10 & 9.484437 & 9.489854 & 9.493750 & 9.484438 \\
& & & & & \\
& & & & & \\
& 0 & 0.501490 & 0.500748 & 0.501500 & 0.501490 \\
& 5 & 5.588750 & 5.588081 & 5.588840 & 5.588750 \\
& 10 & 10.813669 & 10.813058 & 10.813814 & 10.813668 \\
& & & & & \\
& 0 & 0.503687 & 0.501862 & 0.503746 & 0.503687 \\
& 5 & 5.712896 & 5.711448 & 5.713323 & 5.712896 \\
\hline
\end{tabular}

and for the two-dimensional model, we shall present a few examples of the calculations that we have done.

In Table II we display the energies of the ground state and of two excited states calculated for several values of $b$, both for the inverted quartic and the stable quartic oscillator. Four different calculations have been carried out at each point: $E_{\text {diag }}$ results from matrix diagonalization (exact value), $E_{\mathrm{sc}}$ is the semiclassical result, $E_{\mathrm{sc}}+\Delta E$ is the semiclassical result with leading quantum corrections, and $E_{q}$ is the value obtained from Heisenberg matrix mechanics. For the latter, the bandwidth was chosen, when feasible, to give results in essential agreement with $E_{\text {diag }}$. This required values of $B=3-5$. Our general expectation is that the semiclassical calculation with quantum corrections will be an improvement over the purely semiclassical calculation. This expectation is borne out with the exception of the value for $b=-0.005, n=10$. Since the maximum of the potential for $b<0$ is at $1 / 16|b|=12.5$ for $b=-0.005$, the $n=10$ level gets sufficiently close to this maximum that the assumptions upon which our derivation of the semiclassical approximation and of the quantum corrections to it are based become suspect. It was to illustrate this point that results for the stable quartic potential were included. Here there is no sign of the difficulty encountered with the inverted potential.

In Fig. 1 we compare, as a function of the coupling constant, semiclassical matrix elements with the two neighbor-

$$
V=1 / 2 x^{2}+b x^{4}
$$

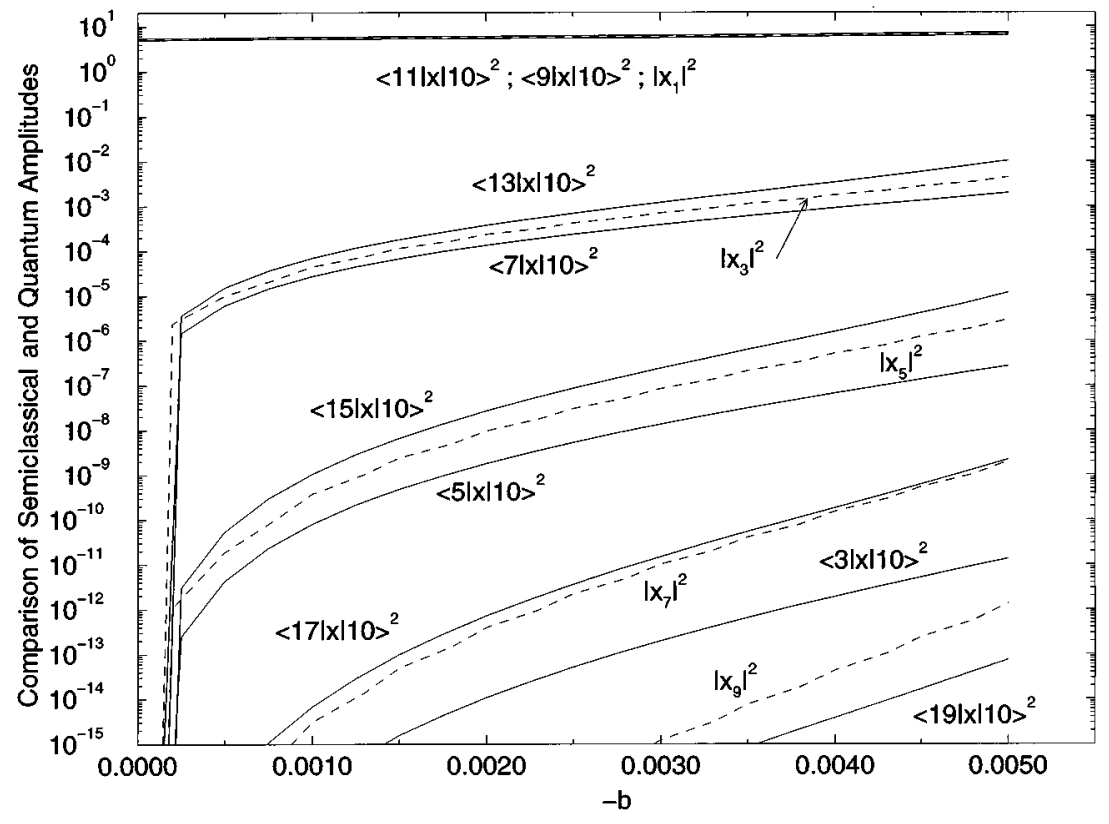

FIG. 1. Comparison of semiclassical matrix elements (Fourier components) for the inverted quartic oscillator and the reference state $n=10$ with the quantum matrix elements that they most closely approximated, as a function of the anharmonicity parameter $b$. The square of the value is plotted with the semiclassical result shown as a dashed line and the associated quantum values as full lines. 
TABLE III. Energies of states labeled by $n_{1}, n_{2}$ for the Hénon-Heiles model defined in the text, as a function of the anharmonicity parameter $b$, for a fixed value of $b^{\prime} . E_{\text {diag }}$ denotes the energy calculated by direct diagonalization, $E_{\mathrm{sc}}$ is the semiclassical approximation, $E_{\mathrm{sc}}+\Delta E$ is the quantum-corrected semiclassical approximation, and $E_{q}$ is the quantum result obtained by the Heisenberg method.

\begin{tabular}{lccccccc}
\hline \hline$b$ & $b^{\prime}$ & $n_{1}$ & $n_{2}$ & $E_{\text {diag }}$ & $E_{\mathrm{sc}}$ & $E_{\mathrm{sc}}+\Delta E$ & $E_{q}$ \\
\hline-0.04 & 0.01 & 0 & 0 & 0.999628 & 0.999600 & 0.999591 & 0.999628 \\
& & 2 & 2 & 4.989952 & 4.990623 & 4.990931 & 4.989952 \\
& & 4 & 4 & 8.967166 & 8.967137 & 8.966849 & 8.967164 \\
-0.08 & 0.01 & 0 & 0 & 0.998504 & 0.998390 & 1.000118 & 0.998504 \\
& & 2 & 2 & 4.958779 & 4.958665 & 4.957205 & 4.958780 \\
& & 4 & 8.862238 & 8.862127 & 8.853510 & 8.862098 \\
\hline \hline
\end{tabular}

ing quantum matrix elements of which it should be the approximate average. (The notation makes it clear that these results are for the reference state $n=10$.) The expectation is well satisfied for the largest matrix elements. We also see that except for the fundamental Fourier component, all other matrix elements are rising exponentially with increasing coupling constant, promising an eventual breakdown of the theory.

We turn next to the two-dimensional generalized HénonHeiles model

$$
\begin{gathered}
H=\frac{1}{2}\left(p_{x}^{2}+\lambda x^{2}\right)+\frac{1}{2}\left(p_{y}^{2}+\mu y^{2}\right)+V(x, y), \\
V(x, y)=b y\left(x^{2}+b^{\prime} y^{2}\right),
\end{gathered}
$$

where we choose $\lambda=1.69$ and $\mu=0.49$, in order to stay away from low-order resonances. We shall consider a range of values of $b$ and $b^{\prime}$ in order to expose different physical situations.

In Table III we display a limited number of results analo- gous to those presented in Table II. Here the addition of quantum corrections to the semiclassical result is far from satisfactory, often overshooting the exact value by more than the starting error. An alternative method that is not strictly an expansion in $(1 / n)$ is under investigation. Again, the accuracy of the nonlinear calculations carried out for advertised form of Heisenberg matrix mechanics is to be noted.

In Figs. 2 and 3 we compare semiclassical and quantum matrix elements for the coordinates $x$ and $y$, respectively, in analogy with what is shown in the one-dimensional case. The same general remarks apply here as for that case. Note that the range of parameters does not include those used in Table III.

\section{ADDITIONAL OBSERVATIONS AND SUGGESTIONS CONCERNING THE SEMICLASSICAL AND CLASSICAL ANALYSES}

In this section, we raise a number of issues related to the semiclassical analysis that it may be profitable to explore.

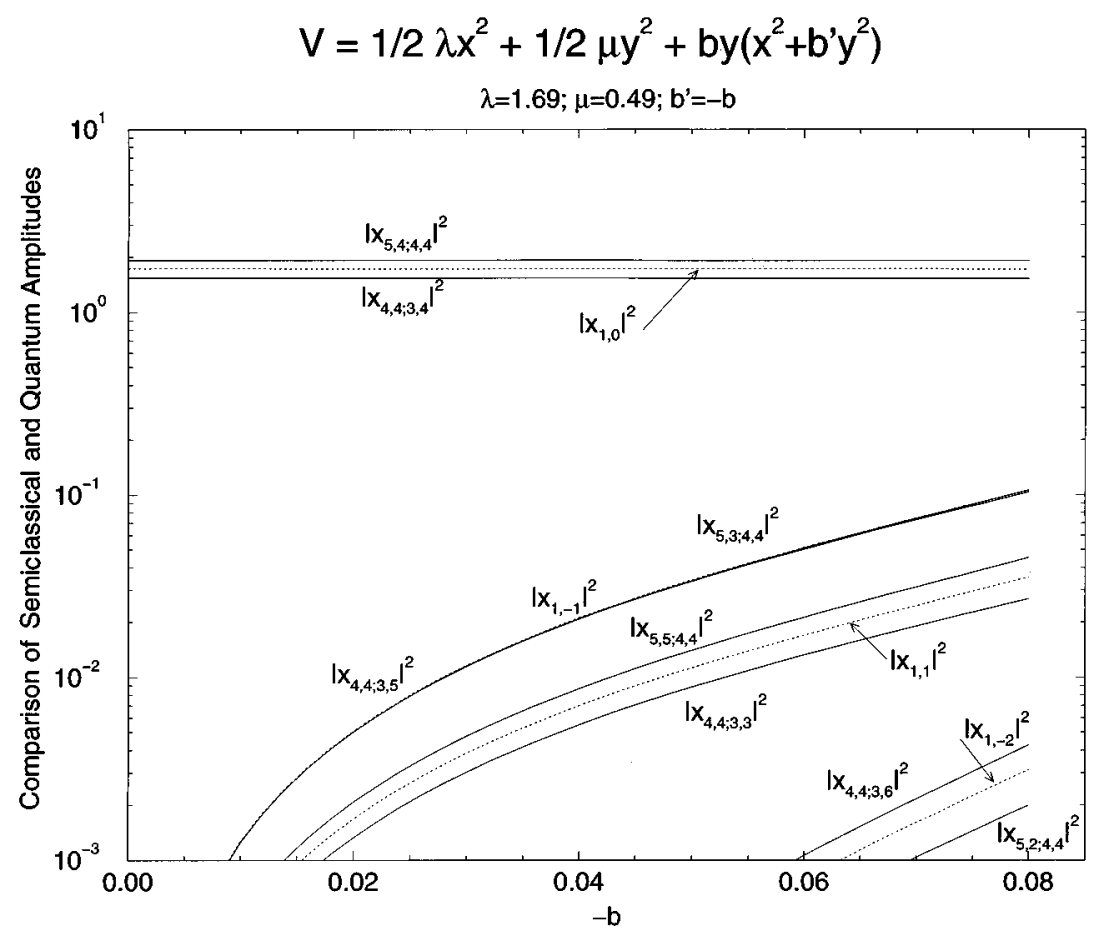

FIG. 2. For the potential shown at the top of the figure, a comparison for the state $\left(n_{1}=n_{2}=4\right)$ of quantum values and of the associated semiclassical approximation for selected matrix elements of the coordinate $x$. The full lines represent the quantum results and the dashed lines the semiclassical values. 


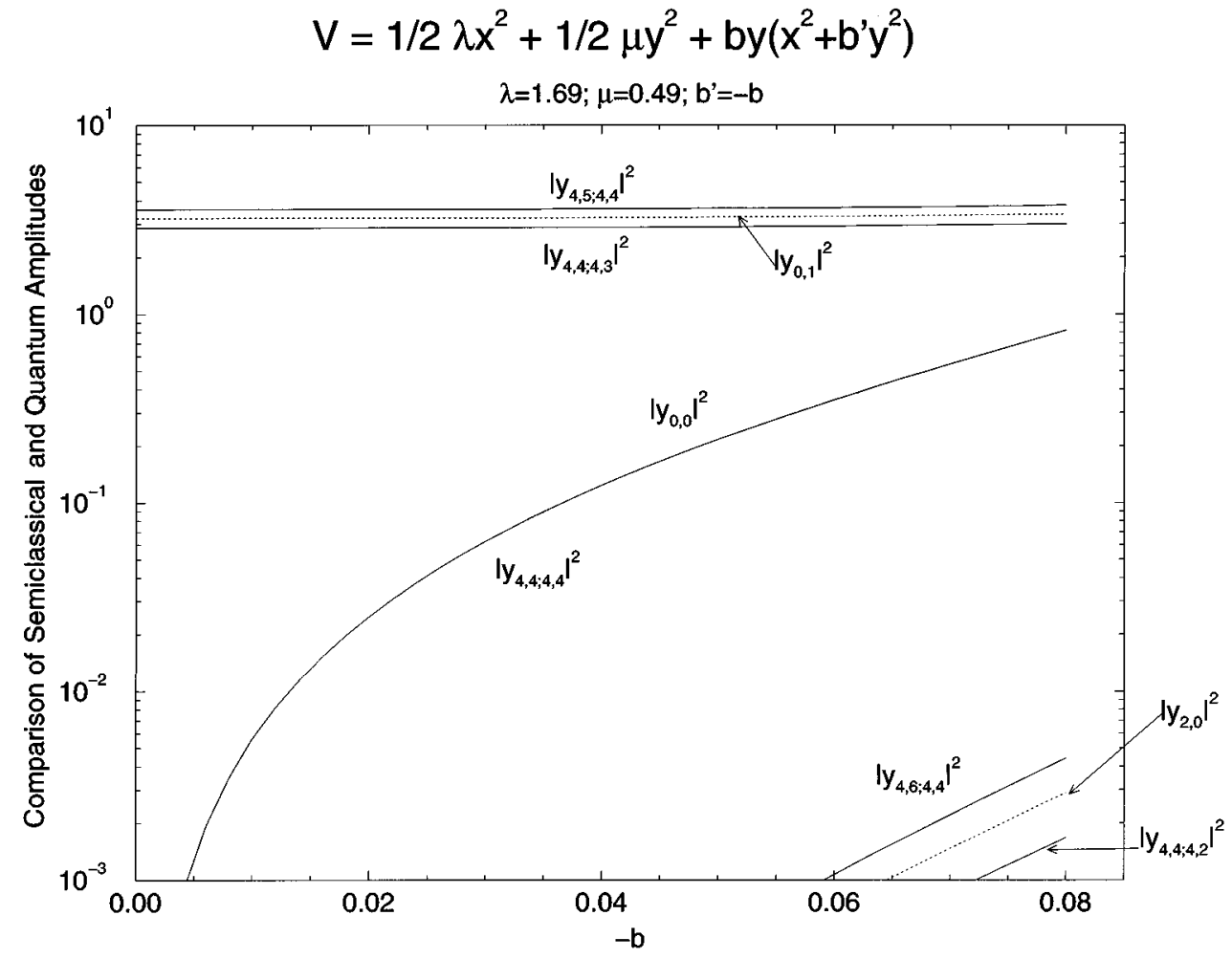

FIG. 3. Same comparison as in Fig. 2, for the coordinate $y$.

Since some of the suggestions put forth in this section are speculative; they may also turn out to be worthless.

\section{A. Fixed action quantization program}

We first consider other possible applications of linear variation about the semiclassical equations, as embodied in Eq. (6.28). These equations are a special case of the equations

$$
S_{a, b} \delta z_{b}=s_{a},
$$

which has a number of possible applications, depending on the choice of the source vector $s$. We discuss briefly several such applications.

(i) There is first of all the basic algorithm of the NewtonRaphson method. In this instance, we choose $s_{a}=-\Psi_{a}^{(\nu)}$, corresponding to a $\nu$ th approximation to $z_{a}$, and the matrix $S$ is also known in this approximation. The solution to (7.1) determines the next approximation to $z_{a}$, namely,

$$
z_{a}^{(\nu+1)}=z_{a}^{(\nu)}+\delta z_{a} .
$$

(ii) If the above solution converges, we thereby define a "stability matrix" $S$. As we have already seen, it is this quantity that occurs when we differentiate Eqs. (6.25) and (6.26) with respect to $n_{i}$, leading to Eqs. (6.28) and (6.29). In addition to the application already described in Sec. VI, we can think of several other applications of these equations. We consider only (6.28), but (6.29) could be brought in. For example, they allow us to compute the energy of neighboring states, according to the formula (two-dimensional example)

$E\left(n_{1}+d_{1}, n_{2}+d_{2}\right)=E\left(n_{1}, n_{2}\right)+\omega_{i} d_{i}+\frac{1}{2} \omega_{i j} d_{i} d_{j}$, where $\omega_{i j}=\left(\partial \omega_{i} / \partial n_{j}\right)$. Work found in the literature, as far as we are aware, uses only the linear approximation to this result. As a second application, when we want to calculate solutions for a fixed Hamiltonian and for values of the action neighboring those for which solutions are already known, the solutions of the linearized equations can be used to obtain improved starting values for the Newton-Raphson iteration.

(iii) To obtain solutions for a neighboring Hamiltonian, one can again use a form of (7.1), with yet another driving term easily derived from the structure of the original nonlinear equations.

An important question that should be susceptible to study by the linearized formalism is the relation of an instability of a solution of the nonlinear equations to the eigenvalues of the matrix $S$.

\section{B. Solutions at fixed frequency: Applications}

We now restrict our attention to the solutions of the equations of motion (6.25) for fixed frequency, i.e., we study the purely classical problem, setting aside for the moment the question of how to adjoin a quantization feature. Let us imagine that we are interested in obtaining the Fourier coefficients as functions of the frequency values. There arises the practical problem of how to choose a sensible grid of frequency values. This problem can be solved presumably by perturbation theory for small coupling and we can use a form of the linearized analysis described above to show us how to change frequencies locally. As a first approximation to quantization, if only energies are of interest, we can compute values of the action from the Fourier coefficients and the frequencies and write

$$
\widetilde{J_{i}}=r_{i}+\frac{1}{2},
$$


where we have used the tilde to indicate a nonquantized value, so that $r_{i}$ is not an integer. We can then extrapolate to the nearest quantized values of the energy by using Eq. (7.3). If we want also the Fourier amplitudes for the quantized invariant tori, we have to successively modify the frequencies until they assume values associated with quantized actions. For this purpose we might use the formula

$$
\delta \omega_{i}=\frac{\partial \omega_{i}}{\partial J_{j}} \delta J_{j}=\frac{\partial^{2} H}{\partial J_{i} \partial J_{j}}\left(n_{j}-r_{j}\right) .
$$

The most practical way of calculating the required second derivatives would probably be from a grid of energy values.

We finally note another possibly interesting way of utilizing a grid of fixed frequency solutions to quantize a system. Consider, as a simple example, a two-dimensional nonresonant system. Write the classical Hamiltonian in the normal form

$$
H=\sum a_{m_{1} m_{2}} J_{1}^{m_{1}} J_{2}^{m_{2}}
$$

It would appear at first sight that the number of points on our grid of solution values will determine the number of terms that we can use in this equation, which yields a set of linear inhomogeneous values for the coefficients $a_{m_{1} m_{2}}$. We would then quantize the resulting Hamiltonian by using the EBK quantization conditions or some variant, following the discussion of Ref. [31]. If we consider the usual procedure for constructing a form such as (7.6), however, we would guess that our previous remarks are much too naive. One's ability to carry the expansion to a higher and higher order is contingent upon obtaining a perturbation expansion to the appropriate order. To make contact with methods based on Fourier series, we must note that an analysis can be carried out that informs us which Fourier components must be included to guarantee equivalence to a perturbation expansion up to a prescribed order. The solution of a nonlinear scheme including only these components, at the same time that it contains a selective (and uncontrolled) summation of higher-order terms, is at least perturbatively correct to some controlled order. It is this latter order that would determine how many terms are allowed in the expansion (7.6). To our knowledge this relationship between the methods based on Fourier expansion and those based on normal forms has not been considered previously.

\section{Direct use of the variational principle}

Can one use the variational principle directly to simply solution of the nonlinear equations? Let $\langle L\rangle^{(\nu)}$ be the $\nu$ th approximation to the average Lagrangian. Expanding to first order,

$$
\langle L\rangle=\langle L\rangle^{(\nu)}+\frac{\delta\langle L\rangle^{(\nu)}}{\delta x_{i, k}} \delta x_{i, k}+\frac{\delta\langle L\rangle^{(\nu)}}{\delta \omega_{i}} \delta \omega_{i}
$$

Consider first the fixed frequency case and choose

$$
\delta x_{i, k}=c_{\nu} \frac{\delta\langle L\rangle^{(\nu)}}{\delta x_{i, k}} .
$$

This is useful provided the average Lagrangian is truly an extremum. It would then appear that a suitable choice of the constant $c_{\nu}$ both as to magnitude and sign would move the average Lagrangian to its extreme value. The reason that we must allow the constant to depend on the order of approximation is that we want to guarantee that the retained firstorder correction is larger than the omitted second-order terms. This means that we must start out with conservatively small values of the constant and let it increase toward unity as (if) we approach convergence.

If we carry out a calculation at fixed action, we have to consider the equation

$$
\frac{\delta\langle L\rangle^{(\nu)}}{\delta \omega_{i}}=J_{i}^{(\nu)}=J_{i}
$$

A comparison with (7.7) suggests that we choose

$$
\delta \omega_{j}=b_{j} J_{j} \quad(\text { no sum }) .
$$

From this assumption, the assumption of fixed $J$, and (7.8) we can derive a pair of linear equations for $b_{j}$, namely,

$$
\sum_{j} \frac{\partial J_{i}}{\partial \omega_{j}} J_{j} b_{j}=-\frac{\partial J_{i}}{\partial x_{j, k}} \frac{\delta\langle L\rangle}{\delta x_{j, k}} c .
$$

Here indices indicating the order of approximation are omitted. In the present case, there is no guarantee that the two first-order correction terms in (7.7) are of the same sign and thus the way that convergence may be achieved, if at all, is somewhat more problematic.

\section{CONCLUDING REMARKS}

In this paper we have presented a different view of the transition from Heisenberg matrix mechanics to the theory of invariant tori. We have suggested a number of possible applications of the ideas that were presented mainly in Secs. II, IV, and VII. We have applied the ideas developed in Secs. II and IV to several standard models. The most important feature that has emerged from these applications is that the Heisenberg mechanics can be developed into a quantum calculus that is only modestly more complicated than the semiclassical calculations associated with the theory of invariant tori. This can be done in two ways; either by starting with the semiclassical approximation and building a correction scheme about it or else by constructing a fully quantum scheme starting from the ground state.

If one were now to ask for the most important next step that one could take with the Heisenberg methods, an excellent candidate for an answer would be to produce solutions for a globally chaotic system such as the one studied by Martens et al. [46]. It would also be worthwhile to revisit some old ground. As an example, we might restudy the edges of the regions, as a function of coupling strength and quantized actions, beyond which converged solutions of our equations cannot be found. Though some of this was done in the present work, the issue is somewhat muddied for the model chosen, since it does not, strictly speaking, possess a Hilbert space. There is evidence, based on calculations for the standard mapping $[47,48]$, that this failure represents an 
independent method for studying the disappearance of invariant tori.

Another idea that might be reexamined is that of approximate tori. Reinhardt and associates have championed this idea, which permits EBK quantization to be applied past the point of breakdown of the associated invariant torus. This idea is suggested both by their work on the quantization of normal-form Hamiltonians [29,30] and on adiabatic switching [23]. It reappears in the work of Martens and Ezra $[32,33]$ in that trajectories that appear to be associated with locally chaotic regions show enough well-defined Fourier components so that quantized actions can be calculated. Independently, we realized that this phenomenon would manifest itself in our work as follows: The nonlinear equations underlying our approach yield a solution when only a small number of Fourier components are retained, but the solution blows up when an attempt to add components is made. By contrast, when invariant tori exist, this is signaled by insensitivity of the solution to the addition of Fourier components beyond a fixed number. The finite Fourier sums describe approximate invariant tori in the same general sense, though in a different approximation, as the approximate normalform Hamiltonians.

The transition from the quantum to the classical domain by the methods of this paper presents a different aspect of the study of the consequences of the order in which the two limits $\hbar \rightarrow 0$ and $t \rightarrow \infty$ are taken [49]. For the order studied in this paper, in which the time limit is taken first, it is quite impossible to strictly reach the regime of multiply periodic motion, as has already been pointed out in the body of our work. It may be of interest to to undertake a further study of the equations that can be obtained in this limit. There may also be some connection of these ideas with the idea of approximate tori.

\section{ACKNOWLEDGMENTS}

We are grateful to J.-M. Yuan and M. Vallières for helpful discussions. This work was supported in part by the United States Department of Energy, under Grant No. 40264-5-25351, and by the Republic of China National Science Council, through Contract No. NSC83-0208-M-002016.

\section{APPENDIX: PROOF THAT SOLVING THE EQUATIONS OF MOTION DIAGONALIZES THE HAMILTONIAN}

We prove directly that a solution of the equations of motion and of the commutation relations guarantees that the Hamiltonian has been rendered diagonal. From the equations of motion, we can derive, in a way that is obvious from the right-hand side of what follows, the equation

$$
\begin{gathered}
\sum_{l}\left\{\left(E_{l}-E_{m}\right)^{2}+\left(E_{n}-E_{l}\right)^{2}\right\} x_{m l} x_{l m} \\
=\left(x V^{\prime}+V^{\prime} x\right)_{m n}=2\left(x V^{\prime}\right)_{m n} .
\end{gathered}
$$

From (2.5) we may write

$$
\left(p^{2}\right)_{m n}=-\sum_{l}\left(E_{l}-E_{m}\right)\left(E_{n}-E_{l}\right) x_{m l} x_{l n}
$$

Equations (A1) and (2.5) can be combined in several useful ways. Thus by subtracting twice (2.5) from (A1) we find

$$
\left(E_{n}-E_{m}\right)^{2}\left(x^{2}\right)_{m n}=-2\left(p^{2}\right)_{m n}+2\left(V^{\prime} x\right)_{m n},
$$

which is the matrix element of the equation

$$
\frac{1}{2}\left[\left[x^{2}, H\right], H\right]=-p^{2}+\left(V^{\prime} x\right)_{m n} .
$$

On the other hand, by adding twice (2.5) and (A1), we find the results

$\sum_{l}\left(2 E_{l}-E_{m}-E_{n}\right)^{2} x_{m l} x_{l n}=2\left(p^{2}\right)_{m n}+2 V^{\prime} x$.

Equation (A5) will be used to prove that $H$ is diagonal in conjunction with another relation, which is a further consequence of the equations of motion, namely,

$$
\begin{aligned}
& \left(E_{n}-E_{m}\right) \sum_{l}\left(2 E_{l}-E_{m}-E_{n}\right)^{2} x_{m l} x_{l n} \\
& =-2 i\left(V^{\prime} p+p V^{\prime}\right)_{m n}+2\left(E_{n}-E_{m}\right)\left(V^{\prime} x\right)_{m n} .
\end{aligned}
$$

Since

$$
i\left(V^{\prime} p+p V^{\prime}\right)_{m n}=2[V, H]_{m n}=2\left(E_{n}-E_{m}\right) V_{m n},
$$

we have, finally, for $m \neq n$, in place of (A5),

$\sum_{l}\left(2 E_{l}-E_{m}-E_{n}\right)^{2} x_{m l} x_{l m}=-4 V_{m n}+2\left(V^{\prime} x\right)_{m n}$

Comparing (A4) with (A8), we conclude that

$$
2\left(p^{2}\right)_{m n}+2\left(V^{\prime} x\right)_{m n}=-4 V_{m n}+2\left(V^{\prime} x\right)_{m n},
$$

or

$$
H_{m n}=\frac{1}{2}\left(p^{2}\right)_{m n}+V_{m n}=0, \quad m \neq n .
$$

[1] H. Hasegawa, M. Robnik, and G. Wunner, Prog. Theor. Phys. Suppl. 98, 198 (1989).

[2] J. Müller, J. Burgdörfer, and D. Noid, Phys. Rev. A 45, 1471 (1992).

[3] A. Einstein, Verh. Dtsch. Phys. Ges. 19, 82 (1917).

[4] M. L. Brillouin, J. Phys. (Paris) 7, 353 (1926).

[5] J. B. Keller, Ann. Phys. (N.Y.) 4, 180 (1958).
[6] C. Jaffé and P. Brumer, J. Phys. Chem. 88, 4829 (1982).

[7] C. Jaffé, J. Chem. Phys. 88, 7603 (1988).

[8] C. Jaffé and F. L. Roberts, in Quantum Dynamics of Chaotic Systems, edited by J.-M. Yuan, D. H. Feng, and G. M. Zaslovsky (Gordon and Breach, New York, 1992), p. 177.

[9] J. J. Morehead, Phys. Rev. A 53, 1285 (1996).

[10] W. Eastes and R. A. Marcus, J. Chem. Phys. 61, 4301 (1974). 
[11] D. W. Noid and R. A. Marcus, J. Chem. Phys. 67, 559 (1977).

[12] D. W. Noid, M. L. Koszykowski, and R. A. Marcus, J. Chem. Phys. 71, 2864 (1979).

[13] I. C. Percival, J. Phys. A 7, 794 (1974).

[14] I. C. Percival and N. Pomphrey, Mol. Phys. 31, 97 (1976).

[15] I. C. Percival and N. Pomphrey, J. Phys. B 9, 3131 (1976).

[16] I. C. Percival and N. Pomphrey, Mol. Phys. 35, 649 (1978).

[17] I. C. Percival, Adv. Chem. Phys. 36, 1 (1977).

[18] I. C. Percival, J. Phys. A 12, L57 (1979).

[19] S. M. Colwell, Chem. Phys. 46, 165 (1980).

[20] A. Klein, J. Math. Phys. 19, 292 (1978).

[21] A. Klein and C. T. Li, J. Math. Phys. 20, 572 (1979).

[22] E. A. Solov'ev, Zh. Éksp. Teor. Fiz. 75, 1261 (1978) [Sov. Phys. JETP 48, 635 (1978)].

[23] R. T. Skodje, F. Borondo, and W. P. Reinhardt, J. Chem. Phys. 82, 4611 (1985).

[24] S. Chapman, B. Garrett, and W. H. Miller, J. Chem. Phys. 64, 502 (1976).

[25] N. De Leon and E. J. Heller, J. Chem. Phys. 78, 4005 (1983).

[26] N. De Leon and E. J. Heller, J. Chem. Phys. 81, 5957 (1984).

[27] F. G. Gustavson, Astrophys. J. 71, 670 (1966).

[28] R. T. Swimm and J. B. Delos, J. Chem. Phys. 71, 1706 (1979).

[29] C. Jaffé and W. P. Reinhardt, J. Chem. Phys. 77, 5191 (1982).

[30] R. B. Shirts and W. P. Reinhardt, J. Chem. Phys. 77, 5204 (1982).

[31] L. E. Fried and G. S. Ezra, J. Chem. Phys. 86, 6270 (1987).

[32] C. C. Martens and G. S. Ezra, J. Chem. Phys. 83, 2990 (1985).

[33] C. C. Martens and G. S. Ezra, J. Chem. Phys. 86, 279 (1987).

[34] A. Garcia-Ayllon, C. C. Martens, J. Santamaria, and G. S. Ezra, J. Chem. Phys. 87, 6609 (1987).

[35] G. S. Ezra, C. C. Martens, and L. E. Fried, J. Phys. Chem. 91, 3721 (1987).
[36] A. K. Kerman and A. Klein, Phys. Rev. 132, 1326 (1963).

[37] G. Do Dang, G. J. Dreiss, R. M. Dreizler, A. Klein, and C.-S. Wu, Nucl. Phys. A 114, 501 (1968).

[38] A. Klein, C. T. Li, and M. Vassanji, J. Math. Phys. 21, 2521 (1980).

[39] C. T. Li and A. Klein, Fizika (Zagreb) 22, 67 (1990).

[40] One form of the trace variational principle, a Lagrangian version, is discussed briefly by M. Born and P. Jordan, Z. Phys. 34, 858 (1925), but never recurs in later work. In a somewhat different context, but for related technical reasons, trace variational principles play a fundamental role in the formulation of quarternion quantum mechanics described by S. L. Adler, Quarternion Quantum Mechanics and Quantum Field Theory (Oxford University Press, Oxford, 1995), p. 374.

[41] H. Goldstein, Classical Mechanics, 2nd ed. (Addison-Wesley, Reading, MA, 1980), p. 402.

[42] W. R. Greenberg, A. Klein, and C. T. Li, Phys. Rev. Lett. 64, 1244 (1995).

[43] W. R. Greenberg, A. Klein, and C. T. Li, Phys. Rep. 264, 167 (1996).

[44] C. T. Li, A. Klein, and F. Krejs, Phys. Rev. D 17, 2311 (1975).

[45] This problem was raised but not solved by M. Born, W. Heisenberg, and P. Jordan, Z. Phys. 35, 557 (1926).

[46] C. C. Martens, R. L. Waterland, and W. P. Reinhardt, J. Chem. Phys. 90, 2328 (1989).

[47] I. C. Percival, in Nonlinear Dynamics and the Beam-Beam Interaction, edited by M. Month and J. C. Herrera, AIP Conf. Proc. No. 57 (AIP, New York, 1979), p. 302.

[48] I. C. Percival, Proc. R. Soc. London Ser. A 413, 132 (1987).

[49] M. Berry, in Chaos and Quantum Physics, edited by M.-J. Giannoni, A. Voros, and J. Zinn-Justin (North-Holland, Amsterdam, 1991), p. 251. 